\title{
EVOLUTION OF NON-ISOTHERMAL LANDAU-DE GENNES NEMATIC LIQUID CRYSTALS FLOWS WITH SINGULAR POTENTIAL*
}

\author{
EDUARD FEIREISL ${ }^{\dagger}$, ELISABETTA ROCCA ${ }^{\ddagger}$, GIULIO SCHIMPERNA ${ }^{\S}$, AND \\ ARGHIR ZARNESCU
}

\begin{abstract}
We discuss a $3 D$ model describing the time evolution of nematic liquid crystals in the framework of Landau-de Gennes theory, where the natural physical constraints are enforced by a singular free energy bulk potential proposed by J.M. Ball and A. Majumdar. The thermal effects are present through the component of the free energy that accounts for intermolecular interactions. The model is consistent with the general principles of thermodynamics and mathematically tractable. We identify the a priori estimates for the associated system of evolutionary partial differential equations and construct global-in-time weak solutions for arbitrary physically relevant initial data.
\end{abstract}

Key words. Liquid crystals, global existence of weak solutions, Navier-Stokes equations.

AMS subject classifications. 76A15, 74G25, 35D30, 35Q30.

\section{Introduction}

The main aim of this paper is to derive and analyze a thermodynamically consistent system of evolutionary equations describing the dynamics of nematic liquid crystal flows in $3 D$. We use the abstract thermodynamic framework proposed by Frémond [12] in conjunction with the Beris-Edwards formulation [3] of isothermal liquid crystal hydrodynamics (cf., e.g., [7]). The state of the complex fluid at the time $t$ and spatial position $x$ is described by means of a $Q$-tensor field $\mathbb{Q}=\mathbb{Q}(t, x)$ for the nematic director orientation, the velocity field $\mathbf{u}=\mathbf{u}(t, x)$, and the absolute temperature $\vartheta=\vartheta(t, x)$.

The main characteristic of nematic liquid crystals is the locally preferred orientation of the nematic molecule directors. This can be described by the $Q$-tensors, which are suitably normalized second order moments of the probability distribution function of the molecules. More precisely, if $\mu_{x}$ is a probability measure on the unit sphere $\mathbb{S}^{2}$ which represents the orientation of the molecules at a point $x$ in space, then a $Q$-tensor $\mathbb{Q}(x)$ is a symmetric and traceless $3 \times 3$ matrix defined as

$$
\mathbb{Q}(x)=\int_{\mathbb{S}^{2}}\left(\mathbf{p} \otimes \mathbf{p}-\frac{1}{3} \mathbb{I}\right) \mathrm{d} \mu_{x}(\mathbf{p})
$$

*Received: October 30, 2012; accepted (in revised form): February 24, 2013. Communicated by Chun Liu.

${ }^{\dagger}$ Institute of Mathematics of the Czech Academy of Sciences, Žitná 25, 11567 Praha 1, Czech Republic (feireisl@math.cas.cz).

The work of E.F. was supported by Grant 201/09/0917 of GA ČR, and partially supported by the FP7-IDEAS-ERC-StG \#256872 (EntroPhase).

‡Dipartimento di Matematica "F. Enriques", Università di Milano, Via Saldini 50, 20133 Milano, Italy (elisabetta.rocca@unimi.it).

The work of E.R. was supported by the FP7-IDEAS-ERC-StG \#256872 (EntroPhase).

$\S$ Dipartimento di Matematica, Università di Pavia, Via Ferrata 1, 27100 Pavia, Italy (giusch04@ unipv.it).

The work of G.S. was supported by the MIUR-PRIN Grant 2008ZKHAHN "Phase transitions, hysteresis and multiscaling".

๑ Pevensey III, University of Sussex, Falmer, BN1 9QH, UK (A.Zarnescu@sussex.ac.uk). The work of A.Z. was partially supported by the FP7-IDEAS-ERC-StG \#256872 (EntroPhase). 
and it is supposed to be a crude measure of how the probability measure $\mu_{x}$ deviates from the isotropic measure $\bar{\mu}$, where $d \bar{\mu}=\frac{1}{4 \pi} d A$; see [6]. In the Onsager model (cf. [6], [19]) the probability measure is assumed to be continuous with density $\rho=\rho(\mathbf{p})$. Then

$$
\mathbb{Q}(x)=\int_{\mathbb{S}^{2}}\left(\mathbf{p} \otimes \mathbf{p}-\frac{1}{3} \mathbb{I}\right) \rho(\mathbf{p}) \mathrm{d} \mathbf{p} .
$$

The fact that $\mu_{x}$ is a probability measure imposes a constraint on the eigenvalues of $\mathbb{Q}$, namely that they are bounded between the values $-\frac{1}{3}$ and $\frac{2}{3}$; see [2]. Thus not any traceless $3 \times 3$ matrix is a physical $Q$-tensor, but only those whose eigenvalues are in $\left(-\frac{1}{3}, \frac{2}{3}\right)$. The hydrodynamic models available in the literature do not have, to our knowledge, a natural way of preserving this physical eigenvalue constraint on the traceless and symmetric matrices. One possibility is to use a singular potential, such as the one proposed by Ball and Majumdar [2], that enforces the physical constraints satisfied by the $Q$-tensors; this is the solution we adopt in our model.

The hydrodynamic theory for the $Q$-tensorial isothermal model in the case of regular bulk potential

$$
\psi_{B}(\mathbb{Q})=\frac{a}{2} \operatorname{tr}\left(\mathbb{Q}^{2}\right)-\frac{b}{2} \operatorname{tr}\left(\mathbb{Q}^{3}\right)+\frac{c}{4} \operatorname{tr}^{2}\left(\mathbb{Q}^{2}\right)
$$

has been recently studied in [22] and [23].

Let us notice that in the literature there are few papers dealing with nonisothermal models for liquid crystal dynamics. Two attempts, in the case when the evolution of the director is described by the vectorial director field $\mathbf{d}$ (standing for the preferred orientation of the molecules at any point), were made in [11] and [8]. In particular, in [8] the stretching and rotation effects of the director field induced by the straining of the fluid were considered and the existence of global in time weak solutions was obtained for the corresponding initial boundary value problem. In the present contribution we follow the thermodynamic approach exploited in [8] in order to deal with the tensorial model obtained using a non-isothermal version of the singular bulk potential $f(\mathbb{Q})$ proposed in $[2]$. In the spirit of $[6]$, we include the temperature dependence in the potential assuming that the coupling term in the free energy functional is given by (cf. also [25] and [20])

$$
\psi_{B}(\vartheta, \mathbb{Q})=f(\mathbb{Q})-U(\vartheta) G(\mathbb{Q}) .
$$

Hence, $\psi_{B}$ is the sum of a singular part $f$, independent of temperature $\vartheta$, with a smooth perturbation depending both on $\vartheta$ and on $\mathbb{Q}$. We assume $U$ to be a convex and decreasing function of $\vartheta$ having controlled growth at infinity (cf. (1.6-1.7) below). Actually, in a neighborhood of a characteristic temperature $\vartheta^{*}$ of the crystal the function $U$ can display a linear growth and can change sign at $\vartheta^{*}$, like $U(\vartheta)=\alpha\left(\vartheta^{*}-\right.$ $\vartheta)$. According to $[6]$, the function $G$ can be, e.g., given by $G(\mathbb{Q})=\mathbb{Q}_{i j} \mathbb{Q}_{i j}=\operatorname{tr}\left(\mathbb{Q}^{2}\right)$. Let us notice that in the Ball and Majumdar [2] approach the temperature dependence is of a different type: actually, they assume

$$
\psi_{B}(\vartheta, \mathbb{Q})=\vartheta f(\mathbb{Q})+G(\mathbb{Q}) .
$$

However, in [2] only the stationary case is considered; hence, one can freely divide by $\vartheta$ the expression $\psi_{B}$ in (1.3) and obtain an expression which corresponds to (1.2) at least for values of $\vartheta$ not too distant from the critical temperature $\vartheta^{*}$. It is worth noting that, in the evolutionary setting, dealing with a free energy of the form (1.3) 
would be mathematically more complicated since the coupling occurs in the singular part of the potential; for this reason we expect that weaker analytic results could be proved in that case. We will devote a forthcoming paper to the analysis of the evolutive model with the free energy (1.3).

Comparing the present analysis with the previous paper [8], a major difficulty is provided here by the presence of the singular potential $f$, which must be properly handled by means of convex analysis tools. In addition to that, we consider here a more complicated version of the heat equation involving an explicit dependence of the thermal relaxation coefficient with respect to the $Q$-tensor (cf. (4.7) below). Actually, this choice, which is more realistic from the physical point of view (in particular, it gives rise to an entropy $s$ depending also on $\mathbb{Q}$ and not only on $\vartheta$, cf. (1.40) below), creates a number of additional mathematical difficulties. The key point, which requires some care to be accomplished, is related to the proof of regularity and strict positivity of $\vartheta$ at the approximate level, two properties which are crucial for the purposes of proving the validity of the entropy inequality and of the total energy balance in the framework of weak solutions.

1.1. Landau-de Gennes free energy with the Ball-Majumdar bulk potential. Denote by $R_{\mathrm{sym}, 0}^{3 \times 3}$ the linear space of symmetric traceless $3 \times 3$ real-valued matrices. The Landau-de Gennes free energy takes the form

$$
\mathcal{F}\left(\mathbb{Q}, \nabla_{x} \mathbb{Q}, \vartheta\right)=\frac{1}{2}\left|\nabla_{x} \mathbb{Q}\right|^{2}+\psi_{B}(\vartheta, \mathbb{Q})-\vartheta \log (\vartheta),
$$

where $\mathbb{Q}(x) \in R_{\mathrm{sym}, 0}^{3 \times 3}$ for all $x$ in the smooth domain $\Omega \subset \mathbb{R}^{3}$.

Ball and Majumdar [2] introduced the bulk component of the internal energy functional by means of a singular functional $\psi_{B}=\psi_{B}(\vartheta, \mathbb{Q})$ that, for any fixed temperature $\vartheta$, blows up when at least one of the eigenvalues of $\mathbb{Q}$ approaches the limiting value $-1 / 3$. In particular, the boundedness of the free energy enforces the boundedness of $\mathbb{Q}$ in $L^{\infty}$. Specifically, we set

$$
\psi_{B}(\vartheta, \mathbb{Q})=f(\mathbb{Q})-U(\vartheta) G(\mathbb{Q}) \text { for } \mathbb{Q} \in R_{\mathrm{sym}, 0}^{3 \times 3},
$$

where

$$
\begin{aligned}
& f(\mathbb{Q})=\left\{\begin{array}{l}
\inf _{\rho \in \mathcal{A}_{\mathbb{Q}}} \int_{S^{2}} \rho(\mathbf{p}) \log (\rho(\mathbf{p})) \mathrm{d} \mathbf{p}, \quad \text { if } \lambda_{i}[\mathbb{Q}] \in(-1 / 3,2 / 3), i=1,2,3, \\
\infty, \quad \text { otherwise, }
\end{array}\right. \\
& \mathcal{A}_{\mathbb{Q}}=\left\{\rho: S^{2} \rightarrow[0, \infty) \mid \rho \in L^{1}\left(S^{2}\right), \int_{S^{2}} \rho(\mathbf{p}) \mathrm{d} \mathbf{p}=1 ; \mathbb{Q}=\int_{S^{2}}\left(\mathbf{p} \otimes \mathbf{p}-\frac{1}{3} \mathbb{I}\right) \rho(\mathbf{p}) \mathrm{d} \mathbf{p}\right\} .
\end{aligned}
$$

The function $f$ is the singular component of the bulk potential. Here 'singular' refers to the fact that the domain is not the whole space (while inside the domain the function is in fact smooth). The function $f$ enjoys the following properties that can easily be deduced from [2, Section 3, Proposition 1]:

- $f: R_{\mathrm{sym}, 0}^{3 \times 3} \rightarrow[-K, \infty]$ is convex and lower semi-continuous, with $K \geq 0$.

- The domain of $f$,

$$
\mathcal{D}[f]=\left\{\mathbb{Q} \in R_{\mathrm{sym}, 0}^{3 \times 3} \mid f(\mathbb{Q})<\infty\right\}=\left\{\mathbb{Q} \in R_{\mathrm{sym}, 0}^{3 \times 3} \mid \lambda_{i}[\mathbb{Q}] \in(-1 / 3,2 / 3)\right\},
$$

is an open convex subset of $R_{\mathrm{sym}, 0}^{3 \times 3}$.

- $f$ is smooth in $\mathcal{D}[f]$. 
The potential $G$ characterizes the action of intermolecular forces. In contrast with Ball and Majumdar [2], we suppose the temperature changes act on this component of the bulk potential. Such a hypothesis is quite common in the literature (see for instance [6]); actually, $U$ is typically assumed to change sign at a critical temperature. Here, we assume that there exists a positive constant $c$ such that

$$
\begin{aligned}
& U \in C^{0}[0, \infty) \cap C^{2}(0, \infty), U(0)>0, U^{\prime} \leq 0, U \text { convex in }[0, \infty), \\
& \limsup _{\vartheta \rightarrow \infty} U^{\prime \prime}(\vartheta) \vartheta^{3 / 2}<+\infty, \\
& \left|U^{\prime}(\vartheta)\right| \leq c|\vartheta|^{-1 / 2} \text { for all } \vartheta \in(0, \infty),
\end{aligned}
$$

and

$$
G \in C^{3}\left(R_{\mathrm{sym}, 0}^{3 \times 3}\right), G \geq 0, G(\mathbb{Q})=G\left(R \mathbb{Q} R^{t}\right) \text { for all } R \in S O(3) .
$$

Let us note that the choice $G(\mathbb{Q})=\operatorname{tr}\left(\mathbb{Q}^{2}\right)$ corresponds to that of [6]. Moreover, it is not restricive to assume that $G$ is uniformly bounded together with its first and second derivatives.

1.2. Thermodynamics. In accordance with the general thermodynamical framework of [12], we introduce the set of state variables

$$
E=\left(\mathbb{Q}, \nabla_{x} \mathbb{Q}, \vartheta\right),
$$

together with the dissipative variables

$$
E^{\mathrm{d}}=\left(\varepsilon(\mathbf{u}), \frac{D \mathbb{Q}}{D t}, \nabla_{x} \vartheta\right)
$$

where

$$
\varepsilon(\mathbf{u})=\frac{1}{2}\left(\nabla_{x} \mathbf{u}+\nabla_{x}^{t} \mathbf{u}\right)
$$

is the symmetric velocity gradient, and

$$
\frac{D \mathbb{Q}}{D t} \equiv \partial_{t} \mathbb{Q}+\mathbf{u} \cdot \nabla_{x} \mathbb{Q}-\mathbb{S}\left(\nabla_{x} \mathbf{u}, \mathbb{Q}\right)
$$

is an analogue of the material derivative characterizing the time evolution of the tensor $\mathbb{Q}$, with

$$
\begin{array}{r}
\mathbb{S}\left(\nabla_{x} \mathbf{u}, \mathbb{Q}\right)=(\xi \varepsilon(\mathbf{u})+\omega(\mathbf{u}))\left(\mathbb{Q}+\frac{1}{3} \mathbb{I}\right)+\left(\mathbb{Q}+\frac{1}{3} \mathbb{I}\right)(\xi \varepsilon(\mathbf{u})-\omega(\mathbf{u})) \\
-2 \xi\left(\mathbb{Q}+\frac{1}{3} \mathbb{I}\right)\left(\mathbb{Q}: \nabla_{x} \mathbf{u}\right), \quad \omega(\mathbf{u})=\frac{1}{2}\left(\nabla_{x} \mathbf{u}-\nabla_{x}^{t} \mathbf{u}\right),
\end{array}
$$

where $\xi$ is a fixed scalar parameter measuring the ratio between the rotation and the aligning effects that a shear flow would have over the directors; see Beris and Edwards [3].

The evolution is ruled by the pseudopotential of dissipation $\Phi$ :

$$
\Phi\left(E^{\mathrm{d}}, E\right)=\mu(\vartheta)|\varepsilon(\mathbf{u})|^{2}+I_{0}\left(\operatorname{div}_{x} \mathbf{u}\right)+\frac{\kappa(\vartheta)}{2 \vartheta}\left|\nabla_{x} \vartheta\right|^{2}+\frac{1}{2 \Gamma(\vartheta)}\left|\frac{D \mathbb{Q}}{D t}\right|^{2},
$$


with the shear viscosity coefficient $\mu$, the heat conductivity coefficient $\kappa$, and the collective rotational viscosity coefficient $\Gamma$. The incompressibility of the fluid is formally enforced by the functional $I_{0}$ - the indicator function of the point $\{0\}$,

$$
I_{0}(z)= \begin{cases}0, & \text { if } z=0, \\ +\infty, & \text { otherwise }\end{cases}
$$

Similarly to $U$, the transport coefficients $\mu(\vartheta), \kappa(\vartheta)$, and $\Gamma(\vartheta)$ change with the temperature. For the sake of simplicity, we suppose that

$$
\mu, \kappa, \Gamma \in C^{2}[0, \infty),\left\{\begin{array}{l}
0<\underline{\mu} \leq \mu(\vartheta) \leq \bar{\mu}, \\
0<\underline{\kappa} \leq \kappa(\vartheta) \leq \bar{\kappa}, \\
0<\underline{\Gamma} \leq \Gamma(\vartheta) \leq \bar{\Gamma}
\end{array}\right\} \text { for all } \vartheta \geq 0 .
$$

1.3. Time evolution. We assume that the fluid has a constant density $\varrho$, say $\varrho=1$. Then, in accordance with the general principles developed in Frémond [12, Chapters 2,3], the time evolution of the system is uniquely determined by the choice of the potentials $\mathcal{F}$ and $\Phi$.

1.3.1. Momentum equation. Newton's second law is expressed by means of a modified Navier-Stokes system:

$$
\partial_{t} \mathbf{u}+\operatorname{div}_{x}(\mathbf{u} \otimes \mathbf{u})=\operatorname{div}_{x} \sigma+\mathbf{g},
$$

where $\mathbf{g}$ is a driving force, and $\sigma$ denotes the stress tensor,

$$
\sigma=\sigma^{\mathrm{d}}+\sigma^{\mathrm{nd}}
$$

The dissipative component of the stress reads

$$
\sigma^{\mathrm{d}}=\frac{\partial \Phi}{\partial \varepsilon(\mathbf{u})}=\frac{\mu(\vartheta)}{2}\left(\nabla_{x} \mathbf{u}+\nabla_{x}^{t} \mathbf{u}\right)-p \mathbb{I},
$$

where $p$ is the pressure. We have

$$
-p \in \partial I_{0}\left(\operatorname{div}_{x} \mathbf{u}\right),
$$

yielding the standard incompressibility constraint

$$
\operatorname{div}_{x} \mathbf{u}=0 .
$$

The specific form of $\sigma^{\text {nd }}$ will be derived below.

1.3.2. Entropy production. The heat flux $\mathbf{q}$ can be decomposed as

$$
\mathbf{q}=\mathbf{q}^{\mathrm{d}}+\mathbf{q}^{\mathrm{nd}}
$$

where the dissipative component obeys the standard Fourier law

$$
\mathbf{q}^{\mathrm{d}}=-\vartheta \frac{\partial \Phi}{\partial \nabla_{x} \vartheta}=-\kappa(\vartheta) \nabla_{x} \vartheta
$$

with the associated entropy flux $\mathbf{q}_{e}=\mathbf{q}^{\mathrm{d}} / \vartheta$. 
The energy density tensor is taken to be

$$
\begin{gathered}
\mathbb{B}=\mathbb{B}^{\mathrm{d}}+\mathbb{B}^{\mathrm{nd}}, \\
\mathbb{B}^{\mathrm{d}}=\frac{\partial \Phi}{\partial \frac{D \mathbb{Q}}{D t}}=\frac{1}{\Gamma(\vartheta)} \frac{D \mathbb{Q}}{D t}, \quad \mathbb{B}^{\mathrm{nd}}=\frac{\partial \mathcal{F}}{\partial \mathbb{Q}}=\mathcal{L}\left[\frac{\partial f(\mathbb{Q})}{\partial \mathbb{Q}}\right]-U(\vartheta) \mathcal{L}\left[\frac{\partial G(\mathbb{Q})}{\partial \mathbb{Q}}\right],
\end{gathered}
$$

where

$$
\mathcal{L}[h(\mathbb{Q})]=h(\mathbb{Q})-\frac{1}{3} \operatorname{tr}[h(\mathbb{Q})] \mathbb{I}, \text { for any } h(\mathbb{Q}) \in R_{\mathrm{sym}}^{3 \times 3},
$$

denotes the projection onto the space of traceless tensors. In other words, $\mathbb{B}^{\text {nd }}$ can be seen as the subdifferential of $\mathcal{F}$ with respect to $\mathbb{Q}$ in that space.

We state the entropy equation in the form (cf. $[4,5]$ for a complete derivation of this equation)

$$
\begin{aligned}
\partial_{t} s+\operatorname{div}_{x}(s \mathbf{u})-\operatorname{div}_{x} \mathbf{q}_{e} & =\frac{1}{\vartheta}\left(\sigma^{\mathrm{d}}: \varepsilon(\mathbf{u})+\mathbb{B}^{\mathrm{d}}: \frac{D \mathbb{Q}}{D t}+\frac{\kappa(\vartheta)}{\vartheta}\left|\nabla_{x} \vartheta\right|^{2}\right) \\
& =\frac{1}{\vartheta}\left(\mu(\vartheta)|\varepsilon(\mathbf{u})|^{2}+\frac{1}{\Gamma(\vartheta)}\left|\frac{D \mathbb{Q}}{D t}\right|^{2}+\frac{\kappa(\vartheta)}{\vartheta}\left|\nabla_{x} \vartheta\right|^{2}\right) \geq 0,
\end{aligned}
$$

with the entropy

$$
s=-\frac{\partial \mathcal{F}}{\partial \vartheta}=1+\log (\vartheta)+U^{\prime}(\vartheta) G(\mathbb{Q}) .
$$

Note that, in accordance with hypotheses (1.6) and (1.8), the entropy $s$ is an increasing function of the temperature.

1.3.3. $Q$-tensor evolution. The internal energy balance reads

$$
\partial_{t} e+\operatorname{div}_{x}(e \mathbf{u})+\operatorname{div}_{x} \mathbf{q}=\sigma: \nabla_{x} \mathbf{u}+\mathbb{B}: \frac{D \mathbb{Q}}{D t}+\mathbb{Y}: \nabla_{x} \frac{D \mathbb{Q}}{D t},
$$

where

$$
e=\mathcal{F}+\vartheta s=\frac{1}{2}\left|\nabla_{x} \mathbb{Q}\right|^{2}+f(\mathbb{Q})-\left(U(\vartheta)-\vartheta U^{\prime}(\vartheta)\right) G(\mathbb{Q})+\vartheta,
$$

and $\mathbb{Y}$ is the energy flux tensor

$$
\mathbb{Y}=\mathbb{Y}^{\text {nd }}=\frac{\partial \mathcal{F}}{\partial \nabla_{x} \mathbb{Q}}=\nabla_{x} \mathbb{Q} .
$$

For simplicity, we assume here that $\mathbb{Y}$ has no dissipative component (and, correspondingly, that $\Phi$ is independent of $\left.\nabla_{x} \mathbb{Q}_{t}\right)$.

The principle of virtual powers (see Frémond [12, Chapter 2]) yields the time evolution of $\mathbb{Q}$, namely,

$$
\operatorname{div}_{x} \mathbb{Y}=\mathbb{B}
$$

in other words,

$$
\partial_{t} \mathbb{Q}+\operatorname{div}_{x}(\mathbb{Q} \mathbf{u})-\mathbb{S}\left(\nabla_{x} \mathbf{u}, \mathbb{Q}\right)=\Gamma(\vartheta)\left(\Delta \mathbb{Q}-\mathcal{L}\left[\frac{\partial f(\mathbb{Q})}{\partial \mathbb{Q}}\right]+U(\vartheta) \mathcal{L}\left[\frac{\partial G(\mathbb{Q})}{\partial \mathbb{Q}}\right]\right),
$$

where $\mathbb{S}$ is defined in (1.10). It is easy to check that the space $R_{\mathrm{sym}, 0}^{3 \times 3}$ is invariant for solutions of $(1.22)$, specifically $\mathbb{Q}(t, \cdot) \in R_{\mathrm{sym}, 0}^{3 \times 3}$ for any $t \geq 0$ as soon as $\mathbb{Q}(0, \cdot) \in R_{\mathrm{sym}, 0}^{3 \times 3}$. 
1.3.4. Total energy balance. Taking the scalar product of the momentum equation (1.13) with $\mathbf{u}$ and adding the resulting expression to (1.18), we obtain the total energy balance in the form

$$
\begin{aligned}
& \partial_{t}\left(\frac{1}{2}|\mathbf{u}|^{2}+e\right)+\operatorname{div}_{x}\left(\left(\frac{1}{2}|\mathbf{u}|^{2}+e\right) \mathbf{u}\right)+\operatorname{div}_{x} \mathbf{q} \\
= & \operatorname{div}_{x}(\sigma \mathbf{u})+\operatorname{div}_{x}\left(\Gamma(\vartheta) \nabla_{x} \mathbb{Q}:\left(\Delta \mathbb{Q}-\mathcal{L}\left[\frac{\partial f(\mathbb{Q})}{\partial \mathbb{Q}}\right]+U(\vartheta) \mathcal{L}\left[\frac{\partial G(\mathbb{Q})}{\partial \mathbb{Q}}\right]\right)\right)+\mathbf{g} \cdot \mathbf{u} .(1.23)
\end{aligned}
$$

It remains to determine $\mathbf{q}^{\text {nd }}$ and $\sigma^{\text {nd }}$.

To this end, we first multiply the entropy balance (1.16) by $\vartheta$. This gives

$$
\vartheta s_{t}-s \mathbf{u} \cdot \nabla_{x} \vartheta+\operatorname{div}_{x}(\vartheta s \mathbf{u})+\operatorname{div}_{x} \mathbf{q}^{d}=\sigma^{\mathrm{d}}: \varepsilon(\mathbf{u})+\mathbb{B}^{\mathrm{d}}: \frac{D \mathbb{Q}}{D t} .
$$

Next, using (1.17), (1.19), (1.15), and (1.20), we get

$$
e_{t}=\mathbb{Y}:: \partial_{t} \nabla_{x} \mathbb{Q}+\mathbb{B}^{\text {nd }}: \partial_{t} \mathbb{Q}+\vartheta s_{t},
$$

where :: indicates contraction both with respect to tensor components and to space variables. Moreover,

$$
\begin{aligned}
\operatorname{div}_{x}(e \mathbf{u}) & =\operatorname{div}_{x}((\mathcal{F}+s \vartheta) \mathbf{u}) \\
& =\mathbf{u} \cdot\left(\mathcal{F}_{\vartheta} \nabla_{x} \vartheta+\mathcal{F}_{\mathbb{Q}}: \nabla_{x} \mathbb{Q}+\mathcal{F}_{\nabla_{x} \mathbb{Q}}:: \nabla_{x} \nabla_{x} \mathbb{Q}\right)+\operatorname{div}_{x}(s \vartheta \mathbf{u}) \\
& =-s \mathbf{u} \cdot \nabla_{x} \vartheta+\mathbf{u} \cdot\left(\mathbb{B}^{\text {nd }}: \nabla_{x} \mathbb{Q}\right)+\mathbf{u} \cdot\left(\mathbb{Y}:: \nabla_{x} \nabla_{x} \mathbb{Q}\right)+\operatorname{div}_{x}(s \vartheta \mathbf{u}) .
\end{aligned}
$$

Replacing (1.25-1.26) in (1.18) and using (1.9), we then have

$$
\begin{gathered}
\mathbb{Y}:: \partial_{t} \nabla_{x} \mathbb{Q}+\mathbb{B}^{\text {nd }}: \partial_{t} \mathbb{Q}+\vartheta s_{t}-s \mathbf{u} \cdot \nabla_{x} \vartheta+\mathbf{u} \cdot\left(\mathbb{B}^{\text {nd }}: \nabla_{x} \mathbb{Q}\right) \\
+\mathbf{u} \cdot\left(\mathbb{Y}:: \nabla_{x} \nabla_{x} \mathbb{Q}\right)+\operatorname{div}_{x}(s \vartheta \mathbf{u})+\operatorname{div}_{x} \mathbf{q} \\
=\sigma^{\mathrm{d}}: \nabla_{x} \mathbf{u}+\sigma^{\mathrm{nd}}: \nabla_{x} \mathbf{u}+\mathbb{B}^{\mathrm{d}}: \frac{D \mathbb{Q}}{D t}+\mathbb{B}^{\mathrm{nd}}: \frac{D \mathbb{Q}}{D t}+\mathbb{Y}:: \nabla_{x} \frac{D \mathbb{Q}}{D t} \\
=\sigma^{\mathrm{d}}: \nabla_{x} \mathbf{u}+\sigma^{\mathrm{nd}}: \nabla_{x} \mathbf{u}+\mathbb{B}^{\mathrm{d}}: \frac{D \mathbb{Q}}{D t}+\mathbb{B}^{\mathrm{nd}}:\left(\partial_{t} \mathbb{Q}+\mathbf{u} \cdot \nabla_{x} \mathbb{Q}-\mathbb{S}\left(\nabla_{x} \mathbf{u}, \mathbb{Q}\right)\right) \\
+\mathbb{Y}::\left(\partial_{t} \nabla_{x} \mathbb{Q}+\nabla_{x}\left(\mathbf{u} \cdot \nabla_{x} \mathbb{Q}\right)-\nabla_{x} \mathbb{S}\left(\nabla_{x} \mathbf{u}, \mathbb{Q}\right)\right) .
\end{gathered}
$$

Simplifying some terms and using symmetry of $\sigma^{\mathrm{d}}$, we have more precisely

$$
\begin{gathered}
\vartheta s_{t}-\mathbf{u} s \cdot \nabla_{x} \vartheta+\operatorname{div}_{x}(s \vartheta \mathbf{u})+\operatorname{div}_{x} \mathbf{q}^{\mathrm{d}}+\operatorname{div}_{x} \mathbf{q}^{\mathrm{nd}} \\
=\sigma^{\mathrm{d}}: \varepsilon(\mathbf{u})+\sigma^{\mathrm{nd}}: \nabla_{x} \mathbf{u}+\mathbb{B}^{\mathrm{d}}: \frac{D \mathbb{Q}}{D t}-\mathbb{B}^{\mathrm{nd}}: \mathbb{S}\left(\nabla_{x} \mathbf{u}, \mathbb{Q}\right) \\
+\mathbb{Y}::\left(\nabla_{x} \mathbf{u} \cdot \nabla_{x} \mathbb{Q}\right)-\mathbb{Y}:: \nabla_{x} \mathbb{S}\left(\nabla_{x} \mathbf{u}, \mathbb{Q}\right) .
\end{gathered}
$$

Then, subtracting (1.24) from (1.28) and using (1.15), we arrive at

$$
\begin{aligned}
\operatorname{div}_{x} \mathbf{q}^{\mathrm{nd}}= & \sigma^{\mathrm{nd}}: \nabla_{x} \mathbf{u}-\left(\mathcal{L}\left[\frac{\partial f(\mathbb{Q})}{\partial \mathbb{Q}}\right]-U(\vartheta) \mathcal{L}\left[\frac{\partial G(\mathbb{Q})}{\partial \mathbb{Q}}\right]\right): \mathbb{S}\left(\nabla_{x} \mathbf{u}, \mathbb{Q}\right) \\
& \quad-\nabla_{x} \mathbb{Q}:: \nabla_{x} \mathbb{S}\left(\nabla_{x} \mathbf{u}, \mathbb{Q}\right)+\left(\nabla_{x} \mathbb{Q} \odot \nabla_{x} \mathbb{Q}\right): \nabla_{x} \mathbf{u} \\
= & \sigma^{\mathrm{nd}}: \nabla_{x} \mathbf{u}-\operatorname{div}_{x}\left(\nabla_{x} \mathbb{Q}: \mathbb{S}\left(\nabla_{x} \mathbf{u}, \mathbb{Q}\right)\right)
\end{aligned}
$$




$$
\begin{aligned}
& +\left(\Delta \mathbb{Q}-\mathcal{L}\left[\frac{\partial f(\mathbb{Q})}{\partial \mathbb{Q}}\right]+U(\vartheta) \mathcal{L}\left[\frac{\partial G(\mathbb{Q})}{\partial \mathbb{Q}}\right]\right): \mathbb{S}\left(\nabla_{x} \mathbf{u}, \mathbb{Q}\right) \\
& +\left(\nabla_{x} \mathbb{Q} \odot \nabla_{x} \mathbb{Q}\right): \nabla_{x} \mathbf{u}
\end{aligned}
$$

Consequently, we deduce that

$$
\mathbf{q}^{\text {nd }}=-\nabla_{x} \mathbb{Q}: \mathbb{S}\left(\nabla_{x} \mathbf{u}, \mathbb{Q}\right),
$$

and

$$
\begin{aligned}
\sigma^{\mathrm{nd}}=\mathbb{Q} \mathbb{H} & -\mathbb{H} \mathbb{Q}+2 \xi[\mathbb{H}: \mathbb{Q}]\left(\mathbb{Q}+\frac{1}{3} \mathbb{I}\right) \\
& -\xi\left[\mathbb{H}\left(\mathbb{Q}+\frac{1}{3} \mathbb{I}\right)+\left(\mathbb{Q}+\frac{1}{3} \mathbb{I}\right) \mathbb{H}\right]-\left(\nabla_{x} \mathbb{Q} \odot \nabla_{x} \mathbb{Q}\right),
\end{aligned}
$$

where we have denoted

$$
\mathbb{H} \equiv \Delta \mathbb{Q}-\mathcal{L}\left[\frac{\partial f(\mathbb{Q})}{\partial \mathbb{Q}}\right]+U(\vartheta) \mathcal{L}\left[\frac{\partial G(\mathbb{Q})}{\partial \mathbb{Q}}\right]
$$

and we have used the identity

$$
\begin{aligned}
& -\mathbb{H}: \mathbb{S}\left(\nabla_{x} \mathbf{u}, \mathbb{Q}\right) \\
& =(\mathbb{Q H}-\mathbb{H} \mathbb{Q}): \nabla_{x} \mathbf{u}+2 \xi(\mathbb{H}: \mathbb{Q})\left(\mathbb{Q}: \nabla_{x} \mathbf{u}\right)-\xi\left[\mathbb{H}\left(\mathbb{Q}+\frac{1}{3} \mathbb{I}\right)+\left(\mathbb{Q}+\frac{1}{3} \mathbb{I}\right) \mathbb{H}\right]: \nabla_{x} \mathbf{u},
\end{aligned}
$$

which holds for any symmetric matrix $\mathbb{H}$.

1.3.5. Evolutionary system. The computations given in the previous section permit us to write the resulting evolutionary system in a concise form:

$$
\begin{aligned}
& \text { INCOMPRESSIBILITY: } \\
& \operatorname{div}_{x} \mathbf{u}=0 \\
& \partial_{t} \mathbf{u}+\operatorname{div}_{x}(\mathbf{u} \otimes \mathbf{u})=\operatorname{div}_{x} \sigma+\mathbf{g} ; \\
& \partial_{t} \mathbb{Q}+\operatorname{div}_{x}(\mathbb{Q} \mathbf{u})-\mathbb{S}\left(\nabla_{x} \mathbf{u}, \mathbb{Q}\right)=\Gamma(\vartheta) \mathbb{H} \\
& \text { TOTAL ENERGy BALANCE: } \\
& \partial_{t}\left(\frac{1}{2}|\mathbf{u}|^{2}+e\right)+\operatorname{div}_{x}\left(\left(\frac{1}{2}|\mathbf{u}|^{2}+e\right) \mathbf{u}\right)+\operatorname{div}_{x} \mathbf{q} \\
& =\operatorname{div}_{x}(\sigma \mathbf{u})+\operatorname{div}_{x}\left(\Gamma(\vartheta) \nabla_{x} \mathbb{Q}: \mathbb{H}\right)+\mathbf{g} \cdot \mathbf{u},
\end{aligned}
$$


with the stress tensor

$$
\begin{aligned}
& \sigma=\mu(\vartheta)\left(\nabla_{x} \mathbf{u}+\nabla_{x}^{t} \mathbf{u}\right)-p \mathbb{I}+2 \xi(\mathbb{H}: \mathbb{Q})\left(\mathbb{Q}+\frac{1}{3} \mathbb{I}\right) \\
&-\xi\left[\mathbb{H}\left(\mathbb{Q}+\frac{1}{3} \mathbb{I}\right)+\left(\mathbb{Q}+\frac{1}{3} \mathbb{I}\right) \mathbb{H}\right]+(\mathbb{Q} \mathbb{H}-\mathbb{H} \mathbb{Q})-\nabla_{x} \mathbb{Q} \odot \nabla_{x} \mathbb{Q},
\end{aligned}
$$

where

$$
\mathbb{H} \equiv \Delta \mathbb{Q}-\mathcal{L}\left[\frac{\partial f(\mathbb{Q})}{\partial \mathbb{Q}}\right]+U(\vartheta) \mathcal{L}\left[\frac{\partial G(\mathbb{Q})}{\partial \mathbb{Q}}\right]
$$

and the internal energy flux

$$
\mathbf{q}=-\kappa(\vartheta) \nabla_{x} \vartheta-\nabla_{x} \mathbb{Q}: \mathbb{S}\left(\nabla_{x} \mathbf{u}, \mathbb{Q}\right)
$$

where

$$
\begin{gathered}
\mathbb{S}\left(\nabla_{x} \mathbf{u}, \mathbb{Q}\right)=(\xi \varepsilon(\mathbf{u})+\omega(\mathbf{u}))\left(\mathbb{Q}+\frac{1}{3} \mathbb{I}\right)+\left(\mathbb{Q}+\frac{1}{3} \mathbb{I}\right)(\xi \varepsilon(\mathbf{u})-\omega(\mathbf{u})) \\
-2 \xi\left(\mathbb{Q}+\frac{1}{3} \mathbb{I}\right)\left(\mathbb{Q}: \nabla_{x} \mathbf{u}\right) .
\end{gathered}
$$

We recall that

$$
e=\frac{1}{2}\left|\nabla_{x} \mathbb{Q}\right|^{2}+f(\mathbb{Q})-\left(U(\vartheta)-\vartheta U^{\prime}(\vartheta)\right) G(\mathbb{Q})+\vartheta, s=1+\log (\vartheta)+U^{\prime}(\vartheta) G(\mathbb{Q}),
$$

where we have anticipated that the relation $\mathcal{L}[\mathbb{Q}]=\mathbb{Q}$ is preserved in the course of evolution.

The system (1.34-1.37) may be supplemented by the entropy inequality

$$
\begin{aligned}
& \partial_{t} s+\operatorname{div}_{x}(s \mathbf{u})-\operatorname{div}_{x}\left(\frac{\kappa(\vartheta)}{\vartheta} \nabla_{x} \vartheta\right) \\
\geq & \frac{1}{\vartheta}\left(\frac{\mu(\vartheta)}{2}\left|\nabla_{x} \mathbf{u}+\nabla_{x}^{t} \mathbf{u}\right|^{2}+\Gamma(\vartheta)|\mathbb{H}|^{2}+\frac{\kappa(\vartheta)}{\vartheta}\left|\nabla_{x} \vartheta\right|^{2}\right) .
\end{aligned}
$$

In order to avoid problems related to the presence of a kinematic boundary, we suppose that the fluid motion is spatially periodic. This can be conveniently formulated by taking the spatial domain $\Omega \subset \mathbb{R}^{3}$ as a flat torus

$$
\Omega=\left(\left.[-\pi, \pi]\right|_{\{-\pi, \pi\}}\right)^{3}
$$

Note also that the pressure $p$ appears explicitly in the energy balance (1.37), in particular, $p$ must be determined from the Navier-Stokes system (1.35) by means of the Helmholtz projection. Such a step may involve insurmountable difficulties in the case of general boundary conditions.

The original state of the system is given by the initial conditions 


$$
\mathbf{u}(0, \cdot)=\mathbf{u}_{0}, \mathbb{Q}(0, \cdot)=\mathbb{Q}_{0}, \vartheta(0, \cdot)=\vartheta_{0}
$$

Our goal in this paper is to study the initial-value problem (1.34-1.37), supplemented with the boundary conditions (1.42) and the initial conditions (1.43), in the framework of weak solutions. As a matter of fact it is the function $\frac{1}{2}|\mathbf{u}|^{2}+e$ that is weakly continuous with respect to time and so the third initial condition in (1.43) should be given in terms of this quantity. In the sequel we will show that, for any choice of finite energy initial data (cf. Section 2 below), the problem possesses a globalin-time weak solution, which additionally satisfies the entropy inequality (1.41) in the sense of distributions. To this end, we first derive formal a priori bounds in order to facilitate the reading of the main (rather technical) part of the proof; see Section 3. The global-in-time weak solutions are constructed as a limit of solutions of a family of approximate problems introduced in Section 4. The most delicate part of the proof is showing strict positivity of the (absolute) temperature by means of a weak variant of the parabolic comparison theorem. The proof of convergence of approximate solutions is completed in Section 5.

\section{Weak solutions, main results}

Weak solutions to the problem (1.34-1.37), (1.42), (1.43) belong to the regularity classes indicated by the a priori bounds discussed in Section 3 below. In particular, we have

$$
\begin{gathered}
\mathbf{u} \in L^{\infty}\left(0, T ; L^{2}\left(\Omega ; \mathbb{R}^{3}\right)\right), \\
\left\{\begin{array}{c}
\mathbb{Q} \in L^{\infty}\left((0, T) \times \Omega ; R_{\mathrm{sym}, 0}^{3 \times 3}\right), \\
\nabla_{x} \mathbb{Q} \in L^{\infty}\left(0, T ; L^{2}\left(\Omega ; R^{27}\right)\right), \\
f(\mathbb{Q}) \in L^{\infty}\left(0, T ; L^{1}(\Omega)\right)
\end{array}\right\},\left\{\begin{array}{c}
\vartheta \in L^{\infty}\left(0, T ; L^{1}(\Omega)\right), \\
\frac{1}{2}|\mathbf{u}|^{2}+e \in C_{w}\left([0, T] ; H^{-3}(\Omega)\right), \\
\log (\vartheta) \in L^{\infty}\left(0, T ; L^{1}(\Omega)\right)
\end{array}\right\},
\end{gathered}
$$

and

$$
\nabla_{x} \mathbf{u} \in L^{2}\left((0, T) \times \Omega ; R^{3 \times 3}\right), \mathbb{Q} \in L^{2}\left(0, T ; W^{2,2}\left(\Omega ; R_{\mathrm{sym}, 0}^{3 \times 3}\right)\right), \quad \nabla_{x} \vartheta \in L^{q}\left((0, T) \times \Omega ; R^{3}\right),
$$

for any $q<5 / 3$.

2.1. Weak solutions. The weak solutions are defined in the standard way. Given the anticipated regularity of the velocity field, the incompressibility constraint (1.34) makes sense a.e. in the set $(0, T) \times \Omega$, while the momentum balance (1.35) is replaced by a family of integral identities

$$
\begin{aligned}
& \int_{0}^{T} \int_{\Omega}\left[\mathbf{u} \cdot \partial_{t} \varphi+(\mathbf{u} \otimes \mathbf{u}): \nabla_{x} \varphi\right] \mathrm{d} x \mathrm{~d} t \\
= & \int_{0}^{T} \int_{\Omega}\left(\sigma: \nabla_{x} \varphi-\mathbf{g} \cdot \varphi\right) \mathrm{d} x \mathrm{~d} t-\int_{\Omega} \mathbf{u}_{0} \cdot \varphi(0, \cdot) \mathrm{d} x
\end{aligned}
$$


satisfied for any test function $\varphi \in C_{c}^{\infty}\left([0, T) \times \Omega ; R^{3}\right)$, and where $\sigma$ is defined in (1.38). Here and in what follows, we always tacitly assume that all quantities appearing under the integrals are (at least) summable in $(0, T) \times \Omega$.

Similarly, the evolutionary equation (1.36) for the $Q$-tensor is replaced by

$$
\begin{aligned}
& \int_{0}^{T} \int_{\Omega}\left[\mathbb{Q}: \partial_{t} \varphi+[\mathbf{u} \mathbb{Q}]: \nabla_{x} \varphi+\mathbb{S}\left(\nabla_{x} \mathbf{u}, \mathbb{Q}\right): \varphi\right] \mathrm{d} x \mathrm{~d} t \\
= & -\int_{0}^{T} \int_{\Omega} \Gamma(\vartheta) \mathbb{H}: \varphi \mathrm{d} x \mathrm{~d} t-\int_{\Omega} \mathbb{Q}_{0}: \varphi(0, \cdot) \mathrm{d} x
\end{aligned}
$$

for any $\varphi \in C_{c}^{\infty}\left([0, T) \times \Omega ; R^{3 \times 3}\right)$, where $\mathbb{S}\left(\nabla_{x} \mathbf{u}, \mathbb{Q}\right)$ is defined in (1.10) and $\mathbb{H}$ in (1.32).

The total energy balance (1.37) is satisfied in the sense of the integral identity

$$
\begin{aligned}
& \int_{0}^{T} \int_{\Omega}\left[\left(\frac{1}{2}|\mathbf{u}|^{2}+e\right) \partial_{t} \varphi+\left(\frac{1}{2}|\mathbf{u}|^{2}+e\right) \mathbf{u} \cdot \nabla_{x} \varphi+\mathbf{q} \cdot \nabla_{x} \varphi\right] \mathrm{d} x \mathrm{~d} t \\
= & \int_{0}^{T} \int_{\Omega}\left[\sigma \mathbf{u} \cdot \nabla_{x} \varphi+\Gamma(\vartheta)\left(\nabla_{x} \mathbb{Q}: \mathbb{H}\right) \cdot \nabla_{x} \varphi-\mathbf{g} \cdot \mathbf{u} \varphi\right] \mathrm{d} x \mathrm{~d} t \\
& -\int_{\Omega}\left(\frac{1}{2}\left|\mathbf{u}_{0}\right|^{2}+e_{0}\right) \varphi(0, \cdot) \mathrm{d} x
\end{aligned}
$$

for any $\varphi \in C_{c}^{\infty}([0, T) \times \Omega)$, where $\mathbf{q}$ is defined in (1.39), $e$ in (1.40), and we have set

$$
e_{0}=\frac{1}{2}\left|\nabla_{x} \mathbb{Q}_{0}\right|^{2}+f\left(\mathbb{Q}_{0}\right)-\left(U\left(\vartheta_{0}\right)-\vartheta_{0} U^{\prime}\left(\vartheta_{0}\right)\right) G\left(\mathbb{Q}_{0}\right)+\vartheta_{0}
$$

2.2. Main result. We are ready to state the main result of this paper.

Theorem 2.1. Let the initial data $\mathbf{u}_{0}, \mathbb{Q}_{0}$, and $\vartheta_{0}$ be given such that

$$
\begin{aligned}
& \mathbf{u}_{0} \in L^{2}\left(\Omega ; R^{3}\right), \operatorname{div}_{x} \mathbf{u}_{0}=0,\left\{\begin{array}{c}
\mathbb{Q}_{0} \in W^{1,2}\left(\Omega ; R_{\mathrm{sym}, 0}^{3 \times 3}\right), \\
f\left(\mathbb{Q}_{0}\right) \in L^{1}(\Omega)
\end{array}\right\}, \\
& \vartheta_{0} \in L^{\infty}(\Omega), \underset{\Omega}{\operatorname{essinf} \vartheta_{0}=\underline{\vartheta}>0 .}
\end{aligned}
$$

Suppose that the functions $U, G$ satisfy the hypotheses (1.6-1.7), (1.8), the transport coefficients $\mu, \kappa$, and $\Gamma$ comply with (1.12), and that

$$
\mathbf{g} \in L^{\infty}\left(0, T ; L^{2}\left(\Omega ; R^{3}\right)\right) .
$$

Then the problem (1.34-1.37), (1.42), (1.43) admits a weak solution $\mathbf{u}, \mathbb{Q}, \vartheta$ in $(0, T) \times \Omega$ in the sense specified in Section 2.1. In addition, there exist positive constants $c$ and $\lambda$ such that

$$
\vartheta(t, \cdot) \geq c \exp (-\lambda t) \underline{\vartheta} \text { for all } t>0
$$

and the entropy inequality (1.41) holds in the sense of distributions.

The rest of the paper is devoted to the proof of Theorem 2.1. For the sake of simplicity, we set $\mathbf{g}=0$ as the proof in the more general case requires only straightforward 
modifications.

\section{A priori bounds}

A priori bounds are natural (formal) constraints imposed on hypothetical smooth solutions by the equations and the initial data.

3.1. Energy bounds. Uniform boundedness in time of the total energy is straightforward consequence of (1.37). In accordance with (2.1), we get

$$
\begin{gathered}
\mathbf{u} \in L^{\infty}\left(0, T ; L^{2}\left(\Omega ; R^{3}\right)\right), \\
f(\mathbb{Q}) \in L^{\infty}\left(0, T ; L^{1}(\Omega)\right), \text { in particular, } \mathbb{Q} \in L^{\infty}\left((0, T) \times \Omega ; R_{\mathrm{sym}, 0}^{3 \times 3}\right), \\
\nabla_{x} \mathbb{Q} \in L^{\infty}\left(0, T ; L^{2}\left(\Omega ; R^{27}\right)\right),
\end{gathered}
$$

and

$$
\vartheta \in L^{\infty}\left(0, T ; L^{1}(\Omega)\right)
$$

where we have anticipated the fact that the absolute temperature is a positive quantity.

3.2. Entropy bounds. Integrating the entropy inequality (1.41) and using (3.3), (1.12)(b) we infer that

$$
\log (\vartheta) \in L^{\infty}\left(0, T ; L^{1}(\Omega)\right) \cap L^{2}\left(0, T ; W^{1,2}(\Omega)\right) .
$$

3.3. Bounds based on energy dissipation. Multiplying the entropy inequality (1.41) by $\vartheta$ we deduce the thermal energy balance in the form

$$
\begin{aligned}
\partial_{t} \vartheta+\mathbf{u} \cdot \nabla_{x} \vartheta-\operatorname{div}_{x}\left(\kappa(\vartheta) \nabla_{x} \vartheta\right) \geq-\vartheta & {\left[\partial_{t}\left(U^{\prime}(\vartheta) G(\mathbb{Q})\right)+\mathbf{u} \cdot \nabla_{x}\left(U^{\prime}(\vartheta) G(\mathbb{Q})\right)\right] } \\
+ & \frac{\mu(\vartheta)}{2}\left|\nabla_{x} \mathbf{u}+\nabla_{x}^{t} \mathbf{u}\right|^{2}+\Gamma(\vartheta) \mathbb{H}: \mathbb{H},
\end{aligned}
$$

where, furthermore,

$$
\vartheta \partial_{t}\left(U^{\prime}(\vartheta) G(\mathbb{Q})\right)=\partial_{t}\left[\left(\vartheta U^{\prime}(\vartheta)-U(\vartheta)+U(0)\right) G(\mathbb{Q})\right]+(U(\vartheta)-U(0)) \partial_{t} G(\mathbb{Q}),
$$

whence

$$
\begin{gathered}
\partial_{t}\left[\left(\vartheta U^{\prime}(\vartheta)-U(\vartheta)+U(0)\right) G(\mathbb{Q})+\vartheta\right] \\
+\mathbf{u} \cdot \nabla_{x}\left[\left(\vartheta U^{\prime}(\vartheta)-U(\vartheta)+U(0)\right) G(\mathbb{Q})+\vartheta\right]-\operatorname{div}_{x}\left(\kappa(\vartheta) \nabla_{x} \vartheta\right) \\
\geq(U(0)-U(\vartheta))\left[\partial_{t} G(\mathbb{Q})+\mathbf{u} \cdot \nabla_{x} G(\mathbb{Q})\right]+\frac{\mu(\vartheta)}{2}\left|\nabla_{x} \mathbf{u}+\nabla_{x}^{t} \mathbf{u}\right|^{2}+\Gamma(\vartheta) \mathbb{H}: \mathbb{H} \\
=(U(0)-U(\vartheta)) \mathcal{L}\left[\frac{\partial G(\mathbb{Q})}{\partial \mathbb{Q}}\right]:\left[\mathbb{S}\left(\nabla_{x} \mathbf{u}, \mathbb{Q}\right)+\Gamma(\vartheta) \mathbb{H}\right] \\
+\frac{\mu(\vartheta)}{2}\left|\nabla_{x} \mathbf{u}+\nabla_{x}^{t} \mathbf{u}\right|^{2}+\Gamma(\vartheta) \mathbb{H}: \mathbb{H} .
\end{gathered}
$$


Since we already know that $\mathbb{Q}$ is uniformly bounded, we deduce from (3.5) that

$$
\begin{aligned}
& \partial_{t} \int_{\Omega}\left[\left(\vartheta U^{\prime}(\vartheta)-U(\vartheta)+U(0)\right) G(\mathbb{Q})+\vartheta\right] \mathrm{d} x \\
\geq & \int_{\Omega}\left[\frac{\mu(\vartheta)}{4}\left|\nabla_{x} \mathbf{u}+\nabla_{x}^{t} \mathbf{u}\right|^{2}+\frac{\Gamma(\vartheta)}{2} \mathbb{H}: \mathbb{H}-C|U(0)-U(\vartheta)|^{2}\right] \mathrm{d} x,
\end{aligned}
$$

where $C>0$ is an explicitly computable constant depending only on $\|\mathbb{Q}\|_{L^{\infty}}, G$, and $\xi$.

By virtue of hypotheses (1.6-1.7), the function $U^{\prime}(\vartheta)$ is bounded, therefore we may combine (3.6) with the energy estimate (3.3), and use assumptions (1.12) and the convexity of $f$ to conclude that

$$
\begin{gathered}
\nabla_{x} \mathbf{u} \in L^{2}\left((0, T) \times \Omega ; R^{3}\right), \mathbb{Q} \in L^{2}\left(0, T ; W^{2,2}\left(\Omega ; R_{\mathrm{sym}, 0}^{3 \times 3}\right)\right), \\
\text { and } \mathcal{L}\left[\frac{\partial f(\mathbb{Q})}{\partial \mathbb{Q}}\right] \in L^{2}\left(0, T ; L^{2}\left(\Omega ; R_{\mathrm{sym}, 0}^{3 \times 3}\right)\right) .
\end{gathered}
$$

Finally, thanks to (3.1-3.2) and (3.7), a comparison of terms in (1.36) gives also

$$
\partial_{t} \mathbb{Q} \in L^{1}\left(0, T ; L^{3}\left(\Omega ; R_{\mathrm{sym}, 0}^{3 \times 3}\right)\right) .
$$

3.4. Bounds on the temperature gradient. The estimates on the temperature gradient are obtained by multiplying (3.5) with $-(1+\vartheta)^{-\alpha}, \alpha>0$. Note that

$$
\frac{1}{(1+\vartheta)^{\alpha}} \operatorname{div}_{x}\left(\kappa(\vartheta) \nabla_{x} \vartheta\right)=\operatorname{div}_{x}\left(\frac{\kappa(\vartheta)}{(1+\vartheta)^{\alpha}} \nabla_{x} \vartheta\right)+\frac{4 \alpha}{(1-\alpha)^{2}} \kappa(\vartheta)\left|\nabla_{x}(1+\vartheta)^{\frac{1-\alpha}{2}}\right|^{2} .
$$

Observe also that the right hand side of (3.5) is uniformly bounded in $L^{1}$ thanks to the previous estimates. Moreover, as we integrate by parts the terms depending on $U$, we obtain, on the right hand side, the terms

$$
G(\mathbb{Q}) \frac{\vartheta}{(1+\vartheta)^{\alpha}} U^{\prime \prime}(\vartheta) \vartheta_{t}+\frac{\vartheta}{(1+\vartheta)^{\alpha}} U^{\prime}(\vartheta) G(\mathbb{Q})_{t},
$$

which we need to control (as well as similar quantities depending on the transport part of the material derivative, which can be treated in the same way). Integrating by parts in time, we get

$$
\partial_{t}\left(G(\mathbb{Q}) Y_{\alpha}(\vartheta)\right)+\left[\frac{\vartheta}{(1+\vartheta)^{\alpha}} U^{\prime}(\vartheta)-Y_{\alpha}(\vartheta)\right] G(\mathbb{Q})_{t},
$$

where

$$
Y_{\alpha}(\vartheta) \equiv \int_{1}^{\vartheta} \frac{s}{(1+s)^{\alpha}} U^{\prime \prime}(s) \mathrm{d} s
$$

and, thanks to (1.6-1.7), the function in square brackets in (3.9) goes like $\vartheta^{1 / 2-\alpha}$ for large $\vartheta$. Hence, due to (3.3), it lies (at least) in $L^{\infty}\left(0, T ; L^{2}(\Omega)\right)$.

Now, using that $G$ is bounded with its first derivatives and recalling (3.8), all terms in (3.9) can be controlled. Hence, after a straightforward manipulation we can conclude that

$$
\nabla_{x}(1+\vartheta)^{\frac{1-\alpha}{2}} \in L^{2}\left((0, T) \times \Omega ; R^{3}\right) \text { for any } \alpha>0
$$


The a priori bounds obtained here are enough to make the weak formulation (2.32.5) meaningful. In particular, the pressure $p$ (cf. (1.38)) can be "computed" directly from (2.3) (cf. [9] and [8] for more details) and it is possible to obtain

$$
p \in L^{5 / 3}((0, T) \times \Omega) .
$$

It can be shown that the a priori bounds obtained in this section are strong enough in order to establish the weak sequential stability of the family of solutions to our problem. However, we do not pursue this path and pass directly to the construction of a family of approximate solutions.

\section{Approximate problems}

The weak solution, the existence of which is claimed in Theorem 2.1, will be constructed by means of a family of approximate problems.

4.1. Approximate velocity fields. The velocity field $\mathbf{u}$ is obtained via the standard Faedo-Galerkin method based on the family of finite dimensional spaces

$$
X_{N}=\left\{\mathbf{v} \in C^{\infty}\left(\Omega ; R^{3}\right) \mid \mathbf{v} \text { is a trigonometric polynomial of order } N, \operatorname{div}_{x} \mathbf{v}=0\right\} .
$$

Accordingly, the momentum equation (2.3) is replaced by a finite system of ordinary differential equations

$$
\begin{aligned}
& \frac{\mathrm{d}}{\mathrm{d} t} \int_{\Omega} \mathbf{u} \cdot \mathbf{v} \mathrm{d} x \\
&=\int_{\Omega}[\mathbf{u}]_{\delta} \otimes \mathbf{u}: \nabla_{x} \mathbf{v} \mathrm{d} x-\delta \int_{\Omega}\left|\nabla_{x} \mathbf{u}\right|^{r-2} \nabla_{x} \mathbf{u}: \nabla_{x} \mathbf{v} \mathrm{d} x-\int_{\Omega} \mu(\vartheta)\left(\nabla_{x} \mathbf{u}+\nabla_{x}^{t} \mathbf{u}\right): \nabla_{x} \mathbf{v} \mathrm{d} x \\
& \quad+\int_{\Omega}\left(\nabla_{x} \mathbb{Q} \odot \nabla_{x} \mathbb{Q}\right): \nabla_{x} \mathbf{v} \mathrm{d} x \\
& \quad-\int_{\Omega}\left\{2 \xi\left(\mathbb{H}_{m, \delta}: \mathbb{Q}\right)\left(\mathbb{Q}+\frac{1}{3} \mathbb{I}\right)-\xi\left[\mathbb{H}_{m, \delta}\left(\mathbb{Q}+\frac{1}{3} \mathbb{I}\right)+\left(\mathbb{Q}+\frac{1}{3} \mathbb{I}\right) \mathbb{H}_{m, \delta}\right]\right\}: \nabla_{x} \mathbf{v} \mathrm{d} x \\
& \quad-\int_{\Omega}\left(\mathbb{Q} \mathbb{H}_{m, \delta}-\mathbb{H}_{m, \delta} \mathbb{Q}\right): \nabla_{x} \mathbf{v} \mathrm{d} x,
\end{aligned}
$$

with the initial condition

$$
\int_{\Omega} \mathbf{u}(0, \cdot) \cdot \mathbf{v} \mathrm{d} x=\int_{\Omega}\left[\mathbf{u}_{0}\right]_{\delta} \cdot \mathbf{v} \mathrm{d} x
$$

for any $\mathbf{v} \in X_{N}$, where

$$
\mathbb{H}_{m, \delta}=\Delta \mathbb{Q}-\mathcal{L}\left[\frac{\partial f_{m}(\mathbb{Q})}{\partial \mathbb{Q}}\right]+U_{\delta}(\vartheta) \mathcal{L}\left[\frac{\partial G(\mathbb{Q})}{\partial \mathbb{Q}}\right] .
$$

Here $r \in(3,10 / 3), \delta$, and $m$ stand for positive parameters; $[\mathbf{u}]_{\delta}$ denotes the standard regularization with respect to the $x$-variable by means of a family of convolutions, while $\left\{f_{m}\right\}_{m>0}$ is a family of smooth convex functions defined on $R_{\mathrm{sym}, 0}^{3 \times 3}$ such that

$f_{m} \leq f$ for all $m \in \mathbb{N}, \quad f_{m_{1}}(\mathbb{Q}) \leq f_{m_{2}}(\mathbb{Q})$ for all $m_{1} \leq m_{2}$, for all $\mathbb{Q} \in R_{\mathrm{sym}, 0}^{3 \times 3}$,

$$
\left\{\begin{array}{c}
f_{m} \rightarrow f, \text { uniformly on compact subsets of } \mathcal{D}[f], \\
\mathcal{L}\left[\frac{\partial f_{m}}{\partial \mathbb{Q}}\right] \rightarrow \mathcal{L}\left[\frac{\partial f}{\partial \mathbb{Q}}\right], \text { uniformly on compact subsets of } \mathcal{D}[f], \\
f_{m} \rightarrow \infty \text { uniformly in } R_{\mathrm{sym}, 0}^{3 \times 3} \backslash \mathcal{D}[f]
\end{array}\right\} \text { as } m \rightarrow \infty,
$$




$$
c_{m}^{1}|\mathbb{Q}|-c_{m}^{2} \leq\left|\mathcal{L}\left[\frac{\partial f_{m}}{\partial \mathbb{Q}}\right]\right| \leq C_{m}^{1}|\mathbb{Q}|+C_{m}^{2} \text { for all } \mathbb{Q} \in R_{\mathrm{sym}, 0}^{3 \times 3}, m>0 .
$$

Notice that the term $\delta\left|\nabla_{x} \mathbf{u}\right|^{r-2} \nabla_{x} \mathbf{u}$ guarantees the additional regularity of $\mathbf{u}$ needed in the $Q$-tensor equation at the approximate level (cf. estimate (4.14) and Section 5.3 below). The restriction on $r$ is essentially of a technical character (cf. Section 5.4 and [8, Section 5] for additional details).

4.2. $Q$-tensors. The equation governing the time evolution of the approximate $Q$-tensors reads

$$
\partial_{t} \mathbb{Q}+\left(\mathbf{u} \cdot \nabla_{x}\right) \mathbb{Q}-\mathbb{S}\left(\nabla_{x} \mathbf{u}, \mathbb{Q}\right)=[\Gamma(\vartheta)]_{\varepsilon}\left(\Delta \mathbb{Q}-\mathcal{L}\left[\frac{\partial f_{m}(\mathbb{Q})}{\partial \mathbb{Q}}\right]+U_{\delta}(\vartheta) \mathcal{L}\left[\frac{\partial G(\mathbb{Q})}{\partial \mathbb{Q}}\right]\right)
$$

with

$$
\mathbb{Q}(0, \cdot)=\left[\mathbb{Q}_{0}\right]_{\delta} .
$$

Here, $[\Gamma(\vartheta)]_{\varepsilon}$ denotes a regularization (via convolutions) of $\Gamma(\vartheta)$ with respect to both $t$ and $x$ variables. Furthermore, $\left[\mathbb{Q}_{0}\right]_{\delta} \in C^{\infty}\left(\Omega ; R_{\mathrm{sym}, 0}^{3 \times 3}\right)$, such that

$$
\left[\mathbb{Q}_{0}\right]_{\delta} \rightarrow \mathbb{Q}_{0} \text { a.e. in } \Omega, f\left(\left[\mathbb{Q}_{0}\right]_{\delta}\right) \rightarrow f\left(\mathbb{Q}_{0}\right) \text { in } L^{1}(\Omega) \text {, as } \delta \rightarrow 0 \text {. }
$$

An explicit construction of $\left[\mathbb{Q}_{0}\right]_{\delta}$ can be obtained, for instance, by truncation and mollification (recall that the domain of $f$ is an open set). Then, the convergence property in (4.6) can be verified using the dominated convergence theorem.

Finally, $U_{\delta}: \mathbb{R} \rightarrow \mathbb{R}$ is a bounded truncation of $U$ satisfying (1.6-1.7) and such that $U_{\delta}^{\prime}(\vartheta)=U_{\delta}^{\prime}(0)$ for $\vartheta \leq 0$.

4.3. Thermal energy balance. The approximate temperature is determined via a "heat" equation of the form

$$
\begin{aligned}
\partial_{t} \vartheta & +\mathbf{u} \cdot \nabla_{x} \vartheta-\operatorname{div}_{x}\left(\kappa(\vartheta) \nabla_{x} \vartheta\right) \\
=-\vartheta \partial_{t} & \left(U_{\delta}^{\prime}(\vartheta) G(\mathbb{Q})\right)-\vartheta \mathbf{u} \cdot \nabla_{x}\left(U_{\delta}^{\prime}(\vartheta) G(\mathbb{Q})\right)+\frac{\mu(\vartheta)}{2}\left|\nabla_{x} \mathbf{u}+\nabla_{x}^{t} \mathbf{u}\right|^{2} \\
& +[\Gamma(\vartheta)]_{\varepsilon} \mathbb{H}_{m, \delta}: \mathbb{H}_{m, \delta}+\delta\left|\nabla_{x} \mathbf{u}\right|^{r},
\end{aligned}
$$

where $\mathbb{H}_{m, \delta}$ is defined in (4.3). Equation (4.7) is complemented with the initial condition

$$
\vartheta(0, \cdot)=\left[\vartheta_{0}\right]_{\delta},
$$

where, similarly to $(4.2),\left[\vartheta_{0}\right]_{\delta}$ denotes a regularization in the space variables.

4.4. Existence of approximate solutions and uniform bounds. Our program for the remaining part of the paper will be to construct approximate solutions to the problem (4.1-4.8) and let successively

$$
m \rightarrow \infty, N \rightarrow \infty, \varepsilon \rightarrow 0 \text {, and, finally, } \delta \rightarrow 0
$$

in order to recover in the limit a weak solution to the problem (1.34-1.37), (1.42-1.43), the existence of which is claimed in Theorem 2.1. 
For fixed values of the parameters $m, N, \varepsilon$, and $\delta$, we can construct local-in-time solutions to the approximate system by means of a Schauder fixed-point argument. This procedure is similar to the one sketched in [8, Section 5] (see also [10, Chapter 6] for further details); hence we leave it to the reader. Moreover, the local solutions can be extended to the whole time interval $[0, T]$ as soon as suitable uniform estimates analogous to the a priori bounds are established. Actually, to simplify notations, we shall directly assume that solutions are defined on the whole $(0, T)$ already in the approximation.

4.4.1. Energy bounds. Bounds on the total energy are obtained in the same way as the a priori bounds. We take $\mathbf{v}=\mathbf{u}(t, \cdot)$ as a test function in (4.1), multiply (4.4) by $-\mathbb{H}_{m, \delta}$, and add the resulting expression to (4.7) to obtain

$$
\frac{\mathrm{d}}{\mathrm{d} t} \int_{\Omega}\left[\frac{1}{2}|\mathbf{u}|^{2}+\frac{1}{2}\left|\nabla_{x} \mathbb{Q}\right|^{2}+f_{m}(\mathbb{Q})-\left(U_{\delta}(\vartheta)-\vartheta U_{\delta}^{\prime}(\vartheta)\right) G(\mathbb{Q})+\vartheta\right] \mathrm{d} x=0 .
$$

We deduce that

$$
\begin{gathered}
\operatorname{ess} \sup _{t \in(0, T)}\|\mathbf{u}(t, \cdot)\|_{L^{2}\left(\Omega ; R^{3}\right)} \leq c, \\
\text { ess } \sup _{t \in(0, T)}\|\mathbb{Q}(t, \cdot)\|_{W^{1,2}\left(\Omega ; R_{\mathrm{sym}, 0}^{3 \times 3}\right)} \leq c,
\end{gathered}
$$

and

$$
\operatorname{ess} \sup _{t \in(0, T)}\|\vartheta(t, \cdot)\|_{L^{1}(\Omega)} \leq c .
$$

In addition, since $\mathbf{u}$ ranges in the finite dimensional space $X_{N}$ consisting of smooth functions, we get

$$
\sup _{t \in[0, T]}\|\mathbf{u}(t, \cdot)\|_{C^{k}\left(\Omega ; R^{3}\right)} \leq c(k, N) \text { for any } k=0,1, \ldots
$$

4.4.2. Bounds on the $Q$-tensors. Since the approximate $Q$-tensors satisfy equation (4.4), where the leading coefficient $[\Gamma(\vartheta)]_{\varepsilon}$ is smooth and the coupling term $\mathbf{u}$. $\nabla_{x} \mathbb{Q}-\mathbb{S}\left(\nabla_{x} \mathbf{u}, \mathbb{Q}\right)$ is regular thanks to $(4.13)$, we may bootstrap the maximal regularity estimates of $L^{q}$-type (see e.g. Krylov [15]) to deduce that

$$
\left\|\partial_{t} \mathbb{Q}\right\|_{L^{q}\left(0, T ; L^{q}\left(\Omega ; R_{\mathrm{sym}, 0}^{3 \times 3}\right)\right)}+\|\mathbb{Q}\|_{L^{q}\left(0, T ; W^{2, q}\left(\Omega ; R_{\mathrm{sym}, 0}^{3 \times 3}\right)\right)} \leq c(q, N, m, \varepsilon, \delta) \text { for any } 1 \leq q<\infty .
$$

Now, we can go back to (4.1) and to use (4.14) to conclude that

$$
\text { ess } \sup _{t \in(0, T)}\left\|\partial_{t} \mathbf{u}(t, \cdot)\right\|_{C^{k}\left(\Omega ; R^{3}\right)} \leq c(k, N, m, \varepsilon, \delta) .
$$

4.4.3. Strict positivity of the absolute temperature. This is one of the most delicate steps in the proof of Theorem 2.1. Our aim is to prove that $\vartheta$ is strictly positive already at the approximate level and uniformly with respect to all approximation parameters. To this aim, we apply the parabolic comparison theorem to equation (4.7) written in the form

$$
\left(1+\vartheta U_{\delta}^{\prime \prime}(\vartheta) G(\mathbb{Q})\right)\left(\partial_{t} \vartheta+\mathbf{u} \cdot \nabla_{x} \vartheta\right)-\operatorname{div}_{x}\left(\kappa(\vartheta) \nabla_{x} \vartheta\right)
$$




$$
\begin{aligned}
&=-\vartheta U_{\delta}^{\prime}(\vartheta) \mathcal{L} {\left[\frac{\partial G(\mathbb{Q})}{\partial \mathbb{Q}}\right]:\left(\mathbb{S}\left(\nabla_{x} \mathbf{u}, \mathbb{Q}\right)+[\Gamma(\vartheta)]_{\varepsilon} \mathbb{H}_{m, \delta}\right)+\frac{\mu(\vartheta)}{2}\left|\nabla_{x} \mathbf{u}+\nabla_{x}^{t} \mathbf{u}\right|^{2} } \\
&+[\Gamma(\vartheta)]_{\varepsilon} \mathbb{H}_{m, \delta}: \mathbb{H}_{m, \delta}+\delta\left|\nabla_{x} \mathbf{u}\right|^{r}
\end{aligned}
$$

Now, a short inspection of (1.10) yields that all terms can be expressed as products of scalar quantities depending on $\mathbb{Q}$ and the symmetric gradient $\varepsilon(\mathbf{u})$ with the only exception of the commutator

$$
\omega(\mathbf{u}) \mathbb{Q}-\mathbb{Q} \omega(\mathbf{u}) .
$$

Fortunately, thanks to assumption (1.8), we have

$$
\mathcal{L}\left[\frac{\partial G(\mathbb{Q})}{\partial \mathbb{Q}}\right]:[\omega(\mathbf{u}) \mathbb{Q}-\mathbb{Q} \omega(\mathbf{u})]=2\left(\mathcal{L}\left[\frac{\partial G(\mathbb{Q})}{\partial \mathbb{Q}}\right] \mathbb{Q}\right): \omega(\mathbf{u})=0
$$

Let us now observe that, by (1.6-1.7), there exists $c>0$ such that $\left|U^{\prime}(\vartheta) \vartheta\right| \leq c \vartheta^{1 / 2}$ for all $\vartheta \geq 0$, and the same inequality can be required to hold for the approximations $U_{\delta}$. Moreover, we can suppose $G$ bounded because the domain of $f$ is bounded and consequently the behavior of $G$ for large $|\mathbb{Q}|$ is not relevant. On account of these considerations, it is not difficult to arrive at

$$
\begin{gathered}
\left(1+\vartheta U_{\delta}^{\prime \prime}(\vartheta) G(\mathbb{Q})\right)\left(\partial_{t} \vartheta+\mathbf{u} \cdot \nabla_{x} \vartheta\right)-\operatorname{div}_{x}\left(\kappa(\vartheta) \nabla_{x} \vartheta\right) \\
\geq-\Lambda \vartheta+\frac{\mu(\vartheta)}{4}\left|\nabla_{x} \mathbf{u}+\nabla_{x}^{t} \mathbf{u}\right|^{2}+\frac{[\Gamma(\vartheta)]_{\varepsilon}}{2} \mathbb{H}_{m, \delta}: \mathbb{H}_{m, \delta}+\delta\left|\nabla_{x} \mathbf{u}\right|^{r},
\end{gathered}
$$

where $\Lambda$ is a positive constant depending only on the structural properties of the functions $U_{\delta}$ and $G$.

Testing (4.16) by $\vartheta^{-}$and using the fact that $U_{\delta}^{\prime \prime}=0$ for $\vartheta<0$, we obtain, by standard arguments, the non-negativity of $\vartheta$.

Next, multiplying equation (4.7) by $\kappa(\vartheta) \partial_{t} \vartheta$ and making use of the available bounds (4.12), (4.13), (4.14), and (1.7), we deduce

$$
\text { ess } \sup _{t \in(0, T)}\|\vartheta\|_{W^{1,2}(\Omega)} \leq c(N, m, \varepsilon, \delta), \quad\left\|\partial_{t} \vartheta\right\|_{L^{2}((0, T) \times \Omega)} \leq c(N, m, \varepsilon, \delta) .
$$

Thus, introducing

$$
\Theta=\mathcal{K}(\vartheta), \mathcal{K}^{\prime}=\kappa, \mathcal{K}(0)=0
$$

and noting that the coefficient $\left(1+\vartheta U_{\delta}^{\prime \prime}(\vartheta) G(\mathbb{Q})\right)$ is uniformly bounded, applying standard elliptic regularity results to (4.16) we arrive at

$$
\|\Theta\|_{L^{2}\left(0, T ; W^{2,2}(\Omega)\right)} \leq c(N, m, \varepsilon, \delta),
$$

whence, by (4.18) and interpolation, it is not difficult to obtain

$$
\|\vartheta\|_{L^{2}\left(0, T ; W^{2,3 / 2}(\Omega)\right)} \leq c(N, m, \varepsilon, \delta) .
$$

Next, we may rewrite (4.17) in the form

$$
a(t, x)\left(\partial_{t} \Theta+\lambda \Theta+\mathbf{u} \cdot \nabla_{x} \Theta\right)-\Delta \Theta=g \geq 0,
$$


where the previous estimates imply that $g \in L^{p}((0, T) \times \Omega)$ for all $p \in[1, \infty)$ and we have set

$$
a=\frac{1+\vartheta U_{\delta}^{\prime \prime}(\vartheta) G(\mathbb{Q})}{\kappa(\vartheta)} \in L^{\infty}((0, T) \times \Omega), a \geq \underline{a}>0,
$$

where $\lambda>0$ depends only on $\Lambda$ and the structural properties of the function $\kappa$, and $\underline{a}$ depends only on $\bar{\kappa}$. For instance, one may take

$$
\lambda \equiv \operatorname{essinf} \frac{\Lambda \vartheta}{\mathcal{K}(\vartheta) a}>0,
$$

and it is worth stressing once more that this choice is independent of all approximation parameters.

Then, multiplying (4.21) by $e^{\lambda t}$ and setting $z \equiv e^{\lambda t} \Theta$, we get

$$
a(t, x)\left(z_{t}+\mathbf{u} \cdot \nabla_{x} z\right)-\Delta z=g e^{\lambda t} \geq 0
$$

whence, testing by $-(z-\underline{\Theta})^{-}$, where $\underline{\Theta}=\mathcal{K}(\underline{\vartheta})$, and integrating by parts the terms with $a$, we obtain the desired conclusion

$$
\vartheta(t, \cdot) \geq c \exp (-\lambda t) \underline{\vartheta} \text { for all } t \in[0, T] .
$$

Let us note that in principle estimate (4.24) holds for smooth $a$ (see e.g. [13]). However, considering the class of solutions specified through (4.18) and (4.20), the procedure can easily be extended to any bounded measurable $a$ by means of density arguments. Indeed, we can consider a smooth (Hölder continuous) approximation $a_{h}$ of $a$ and a smooth approximation $g_{h}$ of $g$, and we can assume $a_{h} \rightarrow a, g_{h} \rightarrow g$ in $L^{p}$ for every $p \in(1, \infty)$. Then, by the standard maximum principle argument (cf. [13]) we can conclude that there exists $\vartheta_{h} \in H^{1}\left(0, T ; L^{2}(\Omega)\right) \cap L^{2}\left(0, T ; W^{2,2}(\Omega)\right)$ (in fact, $\vartheta_{h}$ will be even smoother) satisfying (4.24). Then, also the limit $\widetilde{\vartheta}$ of $\vartheta_{h}$ satisfies the same inequality. This concludes the proof because we have uniqueness of solutions for (4.21), which can be obtained simply testing the difference of the two equations by the time derivative of the difference of two solutions. An analogous proof of the maximum principle argument for (4.21) with bounded measurable $a$ can be found, e.g., in [14, Proposition 3.6, p. 293].

Estimate (4.24) coincides with (2.7) claimed in Theorem 2.1. It is remarkable that (4.24) is independent of the parameters $m, N, \varepsilon$, and $\delta$. Indeed, the choice (4.22) of $\lambda$ is independent of all approximations.

\subsubsection{Moser and regularity estimates on the absolute temperature.}

We prove here that the absolute temperature is (globally in time) Hölder continuous, at least at the approximate level. Although this fact is essentially a consequence of well-known techniques for parabolic equations (see, e.g., [17]), in view of the fact that (4.16) depends on $\vartheta$ in a somehow intricate way we give, for the convenience of the reader, at least the highlights of a direct proof based on Moser iterations.

First of all, we rewrite (4.16) in the form

$$
\left(1+\vartheta U_{\delta}^{\prime \prime}(\vartheta) G(\mathbb{Q})\right)\left(\partial_{t} \vartheta+\mathbf{u} \cdot \nabla_{x} \vartheta\right)-\operatorname{div}_{x}\left(\kappa(\vartheta) \nabla_{x} \vartheta\right)=-\vartheta U_{\delta}^{\prime}(\vartheta) \ell+\nu,
$$

where the functions $\ell$ and $\nu$ collect the various quantities on the right hand side of (4.16) and satisfy

$$
\|\ell\|_{L^{q}\left(0, T ; L^{q}(\Omega)\right)}+\|\nu\|_{L^{q}\left(0, T ; L^{q}(\Omega)\right)} \leq c(q, N, m, \varepsilon, \delta) \text { for any } 1 \leq q<\infty .
$$


Then, by the lower bound (4.24) we are allowed to test (4.26) by $\vartheta^{p-1}$ for a generic $p>1$. Integrating with respect to space variables, we then obtain

$$
\begin{aligned}
& \partial_{t} \int_{\Omega}\left[\left(\frac{1}{p} \vartheta^{p}+H_{\delta, p}(\vartheta) G(\mathbb{Q})\right)+\frac{4(p-1)}{p^{2}} \kappa(\vartheta)\left|\nabla_{x} \vartheta^{p / 2}\right|^{2}\right] \mathrm{d} x \\
= & \int_{\Omega}\left[H_{\delta, p}(\vartheta) \mathcal{L}\left[\frac{\partial G(\mathbb{Q})}{\partial \mathbb{Q}}\right]\left(\partial_{t} \mathbb{Q}+\mathbf{u} \cdot \nabla_{x} \mathbb{Q}\right)-\vartheta^{p} U_{\delta}^{\prime}(\vartheta) \ell+\nu \vartheta^{p-1}\right] \mathrm{d} x,
\end{aligned}
$$

where we have set

$$
H_{\delta, p}(\vartheta):=\int_{1}^{\vartheta} r^{p} U_{\delta}^{\prime \prime}(r) \mathrm{d} r
$$

and we can notice that, due to $(1.6-1.7)$ (or, more precisely, the analogue for $U_{\delta}$ ), we have

$$
H_{\delta, p}(\vartheta) \leq \frac{c}{p}\left(1+\vartheta^{p-1 / 2}\right) .
$$

Then, owing to the fact that $H_{\delta, p}(\vartheta) G(\mathbb{Q})$ is monotone with respect to $\vartheta$ and using the regularity of $\mathbb{Q}$ and $\mathbf{u}$ to estimate the right hand side, we see that (4.27) assumes in fact the structure

$$
\begin{aligned}
& \partial_{t}\left(\|\vartheta\|_{L^{p}(\Omega)}^{p}+p H_{\delta, p}(\vartheta) G(\mathbb{Q})\right)+c_{1}\left\|\nabla_{x} \vartheta^{p / 2}\right\|_{L^{2}\left(\Omega ; R^{3}\right)}^{2} \\
\leq & c_{2}(1+p) \int_{\Omega}\left(\phi \vartheta^{1 / 2}\right)\left(1+\vartheta^{p-1}\right) \mathrm{d} x,
\end{aligned}
$$

where $c_{1}, c_{2}>0$ are absolute constants and $\phi$ collects all the terms depending on $\mathbf{u}$ and $\mathbb{Q}$ (also through $\ell$ and $\nu$ ) and belongs to $L^{q}((0, T) \times \Omega$ ) for all $q \in[1, \infty)$. Thus, for the sake of applying Moser iterations, relation (4.28) shows that we can proceed by working exactly as in the linear case (with forcing term given here by $\phi \vartheta^{1 / 2}$ ). Actually, by (4.18), (4.20), and interpolation we have $\phi \vartheta^{1 / 2} \in L^{s}((0, T) \times \Omega)$ for a suitable $s>3$. Thus, applying the results in [17], we obtain the bound

$$
\|\vartheta\|_{L^{\infty}((0, T) \times \Omega)} \leq c(N, m, \varepsilon, \delta),
$$

and this estimate depends on the approximation parameters, but is uniform with respect to time. Finally, applying the theory developed, e.g., in [16, Section 4], we obtain

$$
\|\vartheta\|_{C^{\alpha}([0, T] \times \Omega)} \leq c(\alpha, N, m, \varepsilon, \delta), \text { for some } \alpha>0,
$$

globally in time.

4.4.5. Estimates based on energy dissipation. The energy bounds analogous to (3.7), (3.10) can be deduced from (4.16) exactly as in Subsection 3.3. Actually, although $\mathbb{Q}$ may not lie in $L^{\infty}$ at this stage, the term

$$
\mathcal{L}\left[\frac{\partial G(\mathbb{Q})}{\partial \mathbb{Q}}\right]: \mathbb{S}\left(\nabla_{x} \mathbf{u}, \mathbb{Q}\right)
$$

does not suffer any summability loss since we can suppose that $G$ has zero gradient when $|\mathbb{Q}|$ is large. Hence, we get

$$
\left\|\nabla_{x} \mathbf{u}\right\|_{L^{2}\left((0, T) \times \Omega ; R^{3 \times 3}\right)} \leq c, \quad \delta^{1 / r}\left\|\nabla_{x} \mathbf{u}\right\|_{L^{r}\left((0, T) \times \Omega ; R^{3 \times 3}\right)} \leq c,
$$




$$
\begin{aligned}
\left\|\mathcal{L}\left[\frac{\partial f_{m}(\mathbb{Q})}{\partial \mathbb{Q}}\right]\right\|_{L^{2}\left((0, T) \times \Omega ; R_{\mathrm{sym}, 0}^{3 \times 3}\right)} & \leq c, \\
\|\mathbb{Q}\|_{L^{2}\left(0, T ; W^{2,2}\left(\Omega ; R_{\mathrm{sym}, 0}^{3 \times 3}\right)\right)} & \leq c,
\end{aligned}
$$

where all constants are independent of the parameters $m, N, \varepsilon$, and $\delta$.

By (4.11), (4.33) and interpolation, we also infer

$$
\|\mathbb{Q}\|_{L^{10}\left((0, T) \times \Omega ; R_{\mathrm{sym}, 0}^{3 \times 3}\right)}+\left\|\nabla_{x} \mathbb{Q}\right\|_{L^{10 / 3}\left((0, T) \times \Omega ; R^{27}\right)} \leq c .
$$

Analogously, from (4.10) and the first (4.31) we get

$$
\|\mathbf{u}\|_{L^{10 / 3}\left((0, T) \times \Omega ; R^{3}\right)} \leq c .
$$

Collecting (4.34), (4.35), and (4.31), we obtain a uniform bound for the coupling terms in (4.4), namely

$$
\left\|\mathbf{u} \cdot \nabla_{x} \mathbb{Q}-\mathbb{S}\left(\nabla_{x} \mathbf{u}, \mathbb{Q}\right)\right\|_{L^{10 / 7}\left((0, T) \times \Omega ; R_{\mathrm{sym}, 0}^{3 \times 3}\right.} \leq c,
$$

whence (4.32-4.33) and a comparison of terms in (4.4) lead to

$$
\left\|\partial_{t} \mathbb{Q}\right\|_{L^{10 / 7}\left((0, T) \times \Omega ; R_{\mathrm{sym}, 0}^{3 \times 3}\right)} \leq c .
$$

We notice however that estimates (4.34-4.37) will be improved in the sequel.

\section{Convergence to the limit system}

The bounds derived in the previous section, being global in time, are sufficient for extending the approximate solutions to the desired existence interval. For this reason, and in order to avoid technicalities, we shall directly assume that solutions are defined over the whole $(0, T)$ already at the approximate level. Our ultimate goal is to perform the limits

$$
m \rightarrow \infty, N \rightarrow \infty, \varepsilon \rightarrow 0 \text {, and, finally, } \delta \rightarrow 0 .
$$

5.1. Uniform bounds on the temperature. Testing (4.16) by $-(1-\vartheta)^{-\alpha}$, $\alpha>0$, it is not difficult to arrive at the analogue of (3.10), namely

$$
\left\|\nabla_{x}(1+\vartheta)^{\frac{1-\alpha}{2}}\right\|_{L^{2}\left(0, T ; L^{2}\left(\Omega ; R^{3}\right)\right)} \leq c, \text { for any } \alpha>0,
$$

Indeed, all terms on the right hand side of (4.16) are uniformly bounded in $L^{2}$. In particular, $\mathcal{L}\left[\frac{\partial G(\mathbb{Q})}{\partial \mathbb{Q}}\right]: \mathbb{S}\left(\nabla_{x} \mathbf{u}, \mathbb{Q}\right)$ has the same regularity of $\nabla_{x} \mathbf{u}$ because, as noted before, we can suppose $G$ to have zero gradient in the set where $|\mathbb{Q}|$ is large. Note also that regularity and strict positivity of (the approximate) $\vartheta$ are essential in order for this procedure to make sense.

Coupling the information coming from (4.12) and (5.1), which are both independent of all approximation parameters, and using interpolation arguments together with the Sobolev imbedding $W^{1,2}(\Omega) \hookrightarrow L^{6}(\Omega)$, we arrive at

$$
\|\vartheta\|_{L^{q}((0, T) \times \Omega)} \leq c \text { for any } 1 \leq q<5 / 3 .
$$

Moreover, another application of interpolation (see also [8, Section 4]) permits us to obtain

$$
\left\|\nabla_{x} \vartheta\right\|_{L^{q}\left((0, T) \times \Omega ; R^{3}\right)} \leq c \text { for any } 1 \leq q<5 / 4 .
$$


Next, thanks to the lower bound established in (4.24) and to the Hölder regularity (4.30), we can (rigorously) divide the heat equation (4.7) by $\vartheta$ to obtain the entropy relation

$$
\begin{aligned}
& \partial_{t}\left(\log (\vartheta)+U_{\delta}^{\prime}(\vartheta) G(\mathbb{Q})\right)+\mathbf{u} \cdot \nabla_{x}\left(\log (\vartheta)+U_{\delta}^{\prime}(\vartheta) G(\mathbb{Q})\right)-\operatorname{div}_{x}\left(\frac{\kappa(\vartheta)}{\vartheta} \nabla_{x} \vartheta\right) \\
= & \frac{1}{\vartheta}\left(\frac{\mu(\vartheta)}{2}\left|\nabla_{x} \mathbf{u}+\nabla_{x}^{t} \mathbf{u}\right|^{2}+\delta\left|\nabla_{x} \mathbf{u}\right|^{r}+[\Gamma(\vartheta)]_{\varepsilon}\left|\mathbb{H}_{m, \delta}\right|^{2}+\frac{\kappa(\vartheta)}{\vartheta}\left|\nabla_{x} \vartheta\right|^{2}\right),
\end{aligned}
$$

which is still an equality at this level.

Integrating (5.4), we deduce that

$$
\text { ess } \sup _{t \in(0, T)}\|\log (\vartheta)\|_{L^{1}(\Omega)} \leq c, \quad\|\log (\vartheta)\|_{L^{2}\left(0, T ; W^{1,2}(\Omega)\right)} \leq c .
$$

Finally, we need an estimate on the time derivative of $\vartheta$. To get this, we go back to (4.7) (or, equivalently, multiply (5.4) by $\vartheta$ ) which we rewrite in the form

$$
\begin{aligned}
& \partial_{t}\left(\vartheta+M_{\delta}(\vartheta) G(\mathbb{Q})\right)+\mathbf{u} \cdot \nabla_{x}\left(\vartheta+M_{\delta}(\vartheta) G(\mathbb{Q})\right)-\operatorname{div}_{x}\left(\kappa(\vartheta) \nabla_{x} \vartheta\right) \\
&=\left(M_{\delta}(\vartheta)-U_{\delta}^{\prime}(\vartheta) \vartheta\right)\left(\partial_{t} G(\mathbb{Q})+\mathbf{u} \cdot \nabla_{x} G(\mathbb{Q})\right) \\
& \quad+\left(\frac{\mu(\vartheta)}{2}\left|\nabla_{x} \mathbf{u}+\nabla_{x}^{t} \mathbf{u}\right|^{2}+\delta\left|\nabla_{x} \mathbf{u}\right|^{r}+[\Gamma(\vartheta)]_{\varepsilon}\left|\mathbb{H}_{m, \delta}\right|^{2}\right),
\end{aligned}
$$

where we have set

$$
M_{\delta}(\vartheta):=\int_{1}^{\vartheta} U_{\delta}^{\prime \prime}(r) r \mathrm{~d} r
$$

Then, testing (5.6) by a test function $\phi \in H^{3}(\Omega)$ and using the previous estimates (4.31-4.35) and (5.2), and the fact that, by $(1.6-1.7), M_{\delta}(\vartheta) \sim \vartheta^{1 / 2}$ for large $\vartheta$, it is easy to check that

$$
\left\|\partial_{t}\left(\vartheta+M_{\delta}(\vartheta) G(\mathbb{Q})\right)\right\|_{L^{1}\left(0, T ; H^{-3}(\Omega)\right)} \leq c,
$$

uniformly with respect to all approximation parameters. On the other hand, a direct calculation based on estimates (5.2), (5.3), and (4.34) permits us to verify that

$$
\left\|\nabla\left(\vartheta+M_{\delta}(\vartheta) G(\mathbb{Q})\right)\right\|_{L^{p}\left((0, T) \times \Omega ; R^{3}\right)} \leq c,
$$

for some $p>1$. Consequently, it is possible to apply the Aubin-Lions compactness lemma to the function $\vartheta+M_{\delta}(\vartheta) G(\mathbb{Q})$. By monotonicity of $M_{\delta}$, smoothness and nonnegativity of $G$, and estimate (5.2), this gives, as a consequence, precompactness of $\vartheta$ in $L^{q}((0, T) \times \Omega)$ for all $q \in[1,5 / 3)$.

5.2. The limit $m \rightarrow \infty$. It is convenient to start with the limit for $m \rightarrow \infty$, with the other parameters $N, \varepsilon$, and $\delta$ fixed. In such a way, the velocity field remains regular at this stage, which facilitates the limit passage considerably. As a result of this step, we obtain that $f(\mathbb{Q})$ lies in $L^{\infty}\left(0, T ; L^{1}(\Omega)\right)$ in the limit. This yields, in particular, uniform boundedness of $\mathbb{Q}$ by a constant independent of the parameters $N, \varepsilon$, and $\delta$. 
We start by recalling that, in accordance with (4.9),

$$
\text { ess } \sup _{t \in(0, T)} \int_{\Omega} f_{m}\left(\mathbb{Q}_{m}\right) \mathrm{d} x \leq c,
$$

whereas, by virtue of the standard identity (see for instance [24])

$$
\mathcal{L}\left[\frac{\partial f_{m}\left(\mathbb{Q}_{m}\right)}{\partial \mathbb{Q}}\right]: \mathbb{Q}_{m}=f_{m}\left(\mathbb{Q}_{m}\right)+f_{m}^{*}\left(\mathcal{L}\left[\frac{\partial f_{m}\left(\mathbb{Q}_{m}\right)}{\partial \mathbb{Q}}\right]\right)
$$

combined with (4.32) and (4.34), we infer that

$$
\left\|f_{m}\left(\mathbb{Q}_{m}\right)\right\|_{L^{5 / 3}((0, T) \times \Omega)} \leq c .
$$

We are ready to perform the limit for $m \rightarrow \infty$. To this end, we denote by $\left\{\mathbf{u}_{m}, \mathbb{Q}_{m}, \vartheta_{m}\right\}_{m>0}$ the family of approximate solutions. First, by virtue of (5.9) and (5.10), we have

$$
f_{m}\left(\mathbb{Q}_{m}\right) \rightarrow \overline{f(\mathbb{Q})} \text { weakly-(*) in } L^{\infty}\left(0, T ; L^{1}(\Omega)\right) \text { and weakly in } L^{5 / 3}((0, T) \times \Omega),
$$

at least for suitable subsequences.

Next, using (4.11), (4.33), (4.34), (4.37), and the Aubin-Lions lemma (cf. $[1,18])$, we get

and

$$
\begin{gathered}
\mathbb{Q}_{m}(t, \cdot) \rightarrow \mathbb{Q}(t, \cdot) \text { in, say, } L^{2}\left(\Omega ; R_{\mathrm{sym}, 0}^{3 \times 3}\right) \text { for a.e. } t \in[0, T], \\
\nabla_{x} \mathbb{Q}_{m} \rightarrow \nabla_{x} \mathbb{Q} \text { (strongly) in } L^{q}\left((0, T) \times \Omega ; R^{27}\right) \text { for all } q \in[1,10 / 3),
\end{gathered}
$$

$$
\Delta \mathbb{Q}_{m} \rightarrow \Delta \mathbb{Q} \text { weakly in } L^{2}\left((0, T) \times \Omega ; R_{\mathrm{sym}, 0}^{3 \times 3}\right) .
$$

Our ultimate goal is to show that

$$
\mathbb{Q}(t, x) \in \mathcal{D}[f] \text { for a.a. }(t, x) \in(0, T) \times \Omega,
$$

yielding, in particular, the desired conclusion

$$
\overline{f(\mathbb{Q})}=f(\mathbb{Q}) .
$$

Using (5.10), the monotonicity of the sequence $f_{m}$, the pointwise convergence of $\left\{\mathbb{Q}_{m}\right\}_{m>0}$, and Fatou's lemma, we obtain

$$
\text { ess } \sup _{t \in(0, T)} \int_{\Omega} f_{m_{0}}(\mathbb{Q}) \mathrm{d} x \leq c \text { for any fixed } m_{0} \text {, with } c \text { independent of } m_{0} ;
$$

whence, by the Levi theorem applied for $m_{0} \rightarrow \infty$, we conclude that

$$
\text { ess } \sup _{t \in(0, T)} \int_{\Omega} f(\mathbb{Q}) \mathrm{d} x \leq c
$$

yielding (5.11).

Let us notice that, as a consequence of (5.11), we have in particular

$$
\|\mathbb{Q}\|_{L^{\infty}\left((0, T) \times \Omega ; R_{\mathrm{sym}, 0}^{3 \times 3}\right)} \leq c .
$$


Recalling (4.33) and applying the standard Gagliardo-Nirenberg inequality (cf. [21, p. 125]), we then also have

$$
\left\|\nabla_{x} \mathbb{Q}\right\|_{L^{4}\left((0, T) \times \Omega ; R^{27}\right)} \leq c,
$$

uniformly in $N, \delta$, and $\varepsilon$. As a consequence, we can also improve (4.36-4.37) as follows:

$$
\begin{gathered}
\left\|\mathbf{u} \cdot \nabla_{x} \mathbb{Q}-\mathbb{S}\left(\nabla_{x} \mathbf{u}, \mathbb{Q}\right)\right\|_{L^{20 / 11}\left((0, T) \times \Omega ; R_{\mathrm{sym}, 0}^{3 \times 3}\right.}^{3 \times 3} \leq c, \\
\left\|\partial_{t} \mathbb{Q}\right\|_{L^{20 / 11}\left((0, T) \times \Omega ; R_{\mathrm{sym}, 0}^{3 \times 3}\right)} \leq c .
\end{gathered}
$$

5.2.1. Strong convergence of the temperature and the limit system. Thanks to the considerations in the last part of Section 5.1, we have

$$
\vartheta_{m} \rightarrow \vartheta \text { in } L^{q}((0, T) \times \Omega) \text { for a certain } q>1 .
$$

Then, the uniform estimates derived above permit us to take the limit $m \rightarrow \infty$ in the momentum equation to obtain

$$
\begin{aligned}
& \frac{\mathrm{d}}{\mathrm{d} t} \int_{\Omega} \mathbf{u} \cdot \mathbf{v} \mathrm{d} x \\
&=\int_{\Omega}[\mathbf{u}]_{\delta} \otimes \mathbf{u}: \nabla_{x} \mathbf{v} \mathrm{d} x-\delta \int_{\Omega}\left|\nabla_{x} \mathbf{u}\right|^{r-2} \nabla_{x} \mathbf{u}: \nabla_{x} \mathbf{v} \mathrm{d} x-\int_{\Omega} \mu(\vartheta)\left(\nabla_{x} \mathbf{u}+\nabla_{x}^{t} \mathbf{u}\right): \nabla_{x} \mathbf{v} \mathrm{d} x \\
& \quad+\int_{\Omega}\left(\nabla_{x} \mathbb{Q} \odot \nabla_{x} \mathbb{Q}\right): \nabla_{x} \mathbf{v} \mathrm{d} x \\
& \quad-\int_{\Omega}\left\{2 \xi\left(\mathbb{H}_{\delta}: \mathbb{Q}\right)\left(\mathbb{Q}+\frac{1}{3} \mathbb{I}\right)-\xi\left[\mathbb{H}_{\delta}\left(\mathbb{Q}+\frac{1}{3} \mathbb{I}\right)+\left(\mathbb{Q}+\frac{1}{3} \mathbb{I}\right) \mathbb{H}_{\delta}\right]\right\}: \nabla_{x} \mathbf{v} \mathrm{d} x \\
& \quad-\int_{\Omega}\left(\mathbb{Q} \mathbb{H}_{\delta}-\mathbb{H}_{\delta} \mathbb{Q}\right): \nabla_{x} \mathbf{v} \mathrm{d} x,
\end{aligned}
$$

for any $\mathbf{v} \in X_{N}$, where

$$
\mathbb{H}_{\delta}=\Delta \mathbb{Q}-\mathcal{L}\left[\frac{\partial f(\mathbb{Q})}{\partial \mathbb{Q}}\right]+U_{\delta}(\vartheta) \mathcal{L}\left[\frac{\partial G(\mathbb{Q})}{\partial \mathbb{Q}}\right]
$$

Analogously, we can take the limit $m \rightarrow \infty$ in the director equation, obtaining

$$
\partial_{t} \mathbb{Q}+\left(\mathbf{u} \cdot \nabla_{x}\right) \mathbb{Q}-\mathbb{S}\left(\nabla_{x} \mathbf{u}, \mathbb{Q}\right)=[\Gamma(\vartheta)]_{\varepsilon} \mathbb{H}_{\delta} .
$$

Actually, we remark that $\mathbf{u}$ is still a smooth vector field in the limit. On the other hand, we cannot prove strong convergence for $\mathbb{H}$ and $\nabla_{x} \vartheta$. For this reason, the heat equation cannot pass to the limit $m \rightarrow \infty$ in the form (4.7). For this reason, it is convenient to replace it by a partial form of the energy balance at this stage.

To do this, for fixed $m$ we multiply (4.4) by $-\mathbb{H}_{m, \delta}$ and sum the result to (4.7). Note that we do not sum the energy contribution coming from the momentum equations at this level. A number of integrations by parts similar to those performed in Section 3.3 then permit us to deduce

$$
\partial_{t}\left(\frac{1}{2}\left|\nabla_{x} \mathbb{Q}_{m}\right|^{2}+f\left(\mathbb{Q}_{m}\right)-\left(U_{\delta}\left(\vartheta_{m}\right)-\vartheta U_{\delta}^{\prime}\left(\vartheta_{m}\right)\right) G\left(\mathbb{Q}_{m}\right)+\vartheta_{m}\right)
$$




$$
\begin{aligned}
&+\operatorname{div}_{x}\left(\mathbf{u}_{m}\left(\frac{1}{2}\left|\nabla_{x} \mathbb{Q}_{m}\right|^{2}+f\left(\mathbb{Q}_{m}\right)-\left(U_{\delta}\left(\vartheta_{m}\right)-\vartheta U_{\delta}^{\prime}\left(\vartheta_{m}\right)\right) G\left(\mathbb{Q}_{m}\right)+\vartheta_{m}\right)\right) \\
&-\operatorname{div}_{x}\left(\nabla_{x} \mathbb{Q}_{m}: \mathbb{S}\left(\nabla_{x} \mathbf{u}_{m}, \mathbb{Q}_{m}\right)\right)-\operatorname{div}_{x}\left(\left[\Gamma\left(\vartheta_{m}\right)\right]_{\varepsilon} \nabla_{x} \mathbb{Q}_{m}: \mathbb{H}_{m, \delta}\right) \\
&-\operatorname{div}_{x}\left(\kappa\left(\vartheta_{m}\right) \nabla_{x} \vartheta_{m}\right)+\mathbb{S}\left(\nabla_{x} \mathbf{u}_{m}, \mathbb{Q}_{m}\right): \mathbb{H}_{m, \delta}+\left(\nabla_{x} \mathbb{Q}_{m} \odot \nabla_{x} \mathbb{Q}_{m}\right): \nabla_{x} \mathbf{u}_{m} \\
&=\frac{\mu\left(\vartheta_{m}\right)}{2}\left|\nabla_{x} \mathbf{u}_{m}+\nabla_{x}^{t} \mathbf{u}_{m}\right|^{2}+\delta\left|\nabla_{x} \mathbf{u}_{m}\right|^{r} .
\end{aligned}
$$

Now it is possible to take the limit $m \rightarrow \infty$ in the above relation. Actually, the worst terms are the quadratic ones on the right hand side. However, they do pass to the limit since the velocity still takes values in the finite dimensional space $X_{N}$ at this level (and, hence, it is a smooth function). We then get

$$
\begin{aligned}
& \partial_{t}(\left.\frac{1}{2}\left|\nabla_{x} \mathbb{Q}\right|^{2}+f(\mathbb{Q})-\left(U_{\delta}(\vartheta)-\vartheta U_{\delta}^{\prime}(\vartheta)\right) G(\mathbb{Q})+\vartheta\right) \\
& \quad+\operatorname{div}_{x}\left(\mathbf{u}\left(\frac{1}{2}\left|\nabla_{x} \mathbb{Q}\right|^{2}+f(\mathbb{Q})-\left(U_{\delta}(\vartheta)-\vartheta U_{\delta}^{\prime}(\vartheta)\right) G(\mathbb{Q})+\vartheta\right)\right) \\
& \quad-\operatorname{div}_{x}\left(\kappa(\vartheta) \nabla_{x} \vartheta\right)-\operatorname{div}_{x}\left(\nabla_{x} \mathbb{Q}: \mathbb{S}\left(\nabla_{x} \mathbf{u}, \mathbb{Q}\right)\right)-\operatorname{div}_{x}\left([\Gamma(\vartheta)]_{\varepsilon} \nabla_{x} \mathbb{Q}: \mathbb{H}_{\delta}\right) \\
& \quad+\mathbb{S}\left(\nabla_{x} \mathbf{u}, \mathbb{Q}\right): \mathbb{H}_{\delta}+\left(\nabla_{x} \mathbb{Q} \odot \nabla_{x} \mathbb{Q}\right): \nabla_{x} \mathbf{u} \\
&=\frac{\mu(\vartheta)}{2}\left|\nabla_{x} \mathbf{u}+\nabla_{x}^{t} \mathbf{u}\right|^{2}+\delta\left|\nabla_{x} \mathbf{u}\right|^{r} .
\end{aligned}
$$

Due to the lack of strong convergence for $\mathbb{H}$ and $\nabla_{x} \vartheta$, the $m$-limit of the entropy equation (5.4) must be be written in the form of an inequality. Indeed, a standard semicontinuity argument yields

$$
\begin{aligned}
& \partial_{t}\left(\log (\vartheta)+U_{\delta}^{\prime}(\vartheta) G(\mathbb{Q})\right)+\mathbf{u} \cdot \nabla_{x}\left(\log (\vartheta)+U_{\delta}^{\prime}(\vartheta) G(\mathbb{Q})\right)-\operatorname{div}_{x}\left(\frac{\kappa(\vartheta)}{\vartheta} \nabla_{x} \vartheta\right) \\
\geq & \frac{1}{\vartheta}\left(\frac{\mu(\vartheta)}{2}\left|\nabla_{x} \mathbf{u}+\nabla_{x}^{t} \mathbf{u}\right|^{2}+\delta\left|\nabla_{x} \mathbf{u}\right|^{r}+[\Gamma(\vartheta)]_{\varepsilon}\left|\mathbb{H}_{\delta}\right|^{2}+\frac{\kappa(\vartheta)}{\vartheta}\left|\nabla_{x} \vartheta\right|^{2}\right)
\end{aligned}
$$

5.3. The limit $N \rightarrow \infty$. Our next goal is to let the Galerkin parameter $N \rightarrow \infty$. In this step, the most difficult points consist in taking the limit of (5.16), in order to let it converge to the Navier-Stokes system, and of the internal energy equation (5.20). To achieve these limits, we need to prove a strong convergence for $\nabla_{x} \mathbf{u}_{N}$. With this purpose, we denote by $\left\{\mathbf{u}_{N}, \mathbb{Q}_{N}, \vartheta_{N}\right\}_{N>0}$ the family of approximate solutions and recall that

$$
\left\|\mathbb{Q}_{N}\right\|_{L^{\infty}\left((0, T) \times \Omega ; R_{\mathrm{sym}, 0}^{3 \times 3}\right)} \leq c
$$

with $c$ independent of $N, \varepsilon$, and $\delta$. Then, we take $\mathbf{v}=\mathbf{u}_{N}$ in (5.16) and correspondingly test (5.18) by $\mathbb{H}_{N}$. Repeating the usual cancellations, we then arrive at

$$
\begin{aligned}
& \partial_{t} \int_{\Omega}\left(\frac{1}{2}\left|\mathbf{u}_{N}\right|^{2}+\frac{1}{2}\left|\nabla_{x} \mathbb{Q}_{N}\right|^{2}+f\left(\mathbb{Q}_{N}\right)\right) \mathrm{d} x \\
& -\int_{\Omega} U_{\delta}\left(\vartheta_{N}\right) \mathcal{L}\left[\frac{\partial G\left(\mathbb{Q}_{N}\right)}{\partial \mathbb{Q}_{N}}\right]:\left(\partial_{t} \mathbb{Q}_{N}+\mathbf{u}_{N} \cdot \nabla_{x} \mathbb{Q}_{N}\right) \mathrm{d} x \\
& +\int_{\Omega}\left(\delta\left|\nabla_{x} \mathbf{u}_{N}\right|^{r}+\frac{\mu\left(\vartheta_{N}\right)}{2}\left|\nabla_{x} \mathbf{u}_{N}+\nabla_{x}^{t} \mathbf{u}_{N}\right|^{2}+\left[\Gamma\left(\vartheta_{N}\right)\right]_{\varepsilon}\left|\mathbb{H}_{N, \delta}\right|^{2}\right) \mathrm{d} x=0
\end{aligned}
$$


where we have used the identities (1.33) and

$$
\int_{\Omega}\left(\mathbf{u}_{N} \cdot \nabla_{x} \mathbb{Q}_{N}\right): \Delta \mathbb{Q}_{N}+\left(\nabla_{x} \mathbb{Q}_{N} \odot \nabla_{x} \mathbb{Q}_{N}\right): \nabla_{x} \mathbf{u}_{N} \mathrm{~d} x=0 .
$$

Next, we integrate (5.23) in time and compute its supremum limit as $N \rightarrow \infty$. We obtain

$$
\begin{aligned}
& \limsup _{N \rightarrow \infty} \int_{0}^{T} \int_{\Omega}\left(\delta\left|\nabla_{x} \mathbf{u}_{N}\right|^{r}+\frac{\mu\left(\vartheta_{N}\right)}{2}\left|\nabla_{x} \mathbf{u}_{N}+\nabla_{x}^{t} \mathbf{u}_{N}\right|^{2}+\left[\Gamma\left(\vartheta_{N}\right)\right]_{\varepsilon}\left|\mathbb{H}_{N, \delta}\right|^{2}\right) \mathrm{d} x \mathrm{~d} t \\
& =\lim _{N \rightarrow \infty} \int_{0}^{T} \int_{\Omega} U_{\delta}\left(\vartheta_{N}\right) \mathcal{L}\left[\frac{\partial G\left(\mathbb{Q}_{N}\right)}{\partial \mathbb{Q}_{N}}\right]:\left(\partial_{t} \mathbb{Q}_{N}+\mathbf{u}_{N} \cdot \nabla_{x} \mathbb{Q}_{N}\right) \mathrm{d} x \mathrm{~d} t \\
& \quad-\liminf _{N \rightarrow \infty} \int_{\Omega}\left(\frac{1}{2}\left|\mathbf{u}_{N}(T)\right|^{2}+\frac{1}{2}\left|\nabla_{x} \mathbb{Q}_{N}(T)\right|^{2}+f\left(\mathbb{Q}_{N}\right)\right) \mathrm{d} x \\
& \quad+\int_{\Omega}\left(\frac{1}{2}\left|\left[\mathbf{u}_{0}\right]_{\delta}\right|^{2}+\frac{1}{2}\left|\nabla_{x}\left[\mathbb{Q}_{0}\right]_{\delta}\right|^{2}+f\left(\mathbb{Q}_{0}\right)\right) \mathrm{d} x \\
& \leq \int_{\Omega} U_{\delta}(\vartheta) \mathcal{L}\left[\frac{\partial G(\mathbb{Q})}{\partial \mathbb{Q}}\right]:\left(\partial_{t} \mathbb{Q}+\mathbf{u} \cdot \nabla_{x} \mathbb{Q}\right) \mathrm{d} x \mathrm{~d} t \\
& \quad-\int_{\Omega}\left(\frac{1}{2}|\mathbf{u}(T)|^{2}+\frac{1}{2}\left|\nabla_{x} \mathbb{Q}_{(T)}\right|^{2}+f(\mathbb{Q})\right) \mathrm{d} x \\
& \quad+\int_{\Omega}\left(\frac{1}{2}\left|\left[\mathbf{u}_{0}\right]_{\delta}\right|^{2}+\frac{1}{2}\left|\nabla_{x}\left[\mathbb{Q}_{0}\right]_{\delta}\right|^{2}+f\left(\mathbb{Q}_{0}\right)\right) \mathrm{d} x
\end{aligned}
$$

Actually, the existence of the limit in the second line above is ensured by the pointwise convergence of $\vartheta_{N}$, by the boundedness of $U_{\delta}$ and $G$ together with their first derivatives, and by estimates (5.2) and (5.14-5.15). Moreover, the liminf on the third line above can be estimated by using semicontinuity of norms with respect to weak convergence.

Now, we observe that it is possible to take the limit $N \rightarrow \infty$ in (5.18). Analogously, we can write the limit of (5.16); however, the second integral on the right hand side has to be temporarily written as

$$
-\delta \int_{\Omega} \overline{\left|\nabla_{x} \mathbf{u}\right|^{r-2} \nabla_{x} \mathbf{u}}: \nabla_{x} \mathbf{v} \mathrm{d} x
$$

in the limit, since strong convergence of $\nabla_{x} \mathbf{u}$ is not achieved yet.

Then, we take $\mathbf{v}=\mathbf{u}$ in the $N$-limit of (5.16) and test the $N$-limit of (5.18) by $\mathbb{H}$. Note that this procedure is rigorous (and gives rise to an equality) thanks to the fact that we have that $\nabla_{x} \mathbf{u}$ is bounded in $L^{r}$, where $r>3$, uniformly in $N$ and, consequently, the coupling term

$$
\mathbf{u} \cdot \nabla_{x} \mathbb{Q}-\mathbb{S}\left(\nabla_{x} \mathbf{u}, \mathbb{Q}\right)
$$

in (5.18) lies in $L^{2}$ even in the limit. Hence, equation (5.18) can still be read as a relation in $L^{2}$ and use of the $L^{2}$-test function $\mathbb{H}$ is consequently permitted (note that this will no longer be true in the limit $\delta \rightarrow 0$ ). This is exactly the reason why the $r$-Laplacean regularization has been added in the momentum equation.

This procedure permits us to achieve, in the limit $N \rightarrow \infty$, the analogue of (5.23) (integrated in time). Comparing with (5.25) we then obtain

$$
\limsup _{N \rightarrow \infty} \int_{0}^{T} \int_{\Omega}\left(\delta\left|\nabla_{x} \mathbf{u}_{N}\right|^{r}+\frac{\mu\left(\vartheta_{N}\right)}{2}\left|\nabla_{x} \mathbf{u}_{N}+\nabla_{x}^{t} \mathbf{u}_{N}\right|^{2}+\left[\Gamma\left(\vartheta_{N}\right)\right]_{\varepsilon}\left|\mathbb{H}_{N, \delta}\right|^{2}\right) \mathrm{d} x \mathrm{~d} t
$$




$$
\leq \int_{0}^{T} \int_{\Omega}\left(\delta \overline{\left.\nabla_{x} \mathbf{u}\right|^{r} \nabla_{x} \mathbf{u}} \cdot \nabla_{x} \mathbf{u}+\frac{\mu(\vartheta)}{2}\left|\nabla_{x} \mathbf{u}+\nabla_{x}^{t} \mathbf{u}\right|^{2}+[\Gamma(\vartheta)]_{\varepsilon}\left|\mathbb{H}_{\delta}\right|^{2}\right) \mathrm{d} x \mathrm{~d} t
$$

whence a simple monotonicity argument permits us to deduce the strong convergence

$$
\nabla_{x} \mathbf{u}_{N} \rightarrow \nabla_{x} \mathbf{u} \text { in } L^{r}\left((0, T) \times \Omega ; R^{9}\right) .
$$

Using this relation together with (5.13), (5.15), and the Aubin-Lions lemma (cf. [1, 18]), we can also take the limit $N \rightarrow \infty$ of (5.20) (and in particular of the quadratic terms on the right hand side).

Moreover, (5.28) permits us to identify the limit of $\left|\nabla_{x} \mathbf{u}\right|^{r-2} \nabla_{x} \mathbf{u}$ in the momentum equation (cf. (5.26)). In particular, the Navier-Stokes system (5.16) passes to the desired limit as $N \rightarrow \infty$.

Next, we notice that the (infimum) limit $N \rightarrow \infty$ can be taken in the entropy inequality (5.21) as in the previous section. Thus, to complete the passage to the limit with respect to $N \rightarrow \infty$ it is sufficient to recover the total energy balance (cf. (1.37)). With this aim, we can notice that, in the limit $N \rightarrow \infty,(5.16)$ is no longer a relation in the finite-dimensional space $X_{N}$, but can rather be interpreted as a true PDE, (at least) in the distributional sense. In other words, we can rewrite it in the form

$$
\partial_{t} \mathbf{u}+\operatorname{div}_{x}\left([\mathbf{u}]_{\delta} \otimes \mathbf{u}\right)=\operatorname{div}_{x} \sigma
$$

where

$$
\begin{aligned}
\sigma=\mu(\vartheta) & \left(\nabla_{x} \mathbf{u}+\nabla_{x}^{t} \mathbf{u}\right)+\delta\left|\nabla_{x} \mathbf{u}\right|^{r-2} \nabla_{x} \mathbf{u}-p \mathbb{I} \\
& +2 \xi\left(\mathbb{H}_{\delta}: \mathbb{Q}\right)\left(\mathbb{Q}+\frac{1}{3} \mathbb{I}\right)-\xi\left[\mathbb{H}_{\delta}\left(\mathbb{Q}+\frac{1}{3} \mathbb{I}\right)+\left(\mathbb{Q}+\frac{1}{3} \mathbb{I}\right) \mathbb{H}_{\delta}\right] \\
& +\left(\mathbb{Q H}_{\delta}-\mathbb{H}_{\delta} \mathbb{Q}\right)-\nabla_{x} \mathbb{Q} \odot \nabla_{x} \mathbb{Q} .
\end{aligned}
$$

Then, multiplying (5.29) by $\mathbf{u}$ and adding the result to (5.20), standard integrations by parts permits us to get the total energy balance (1.37) (where of course, at this level, the stress $\sigma$ still contains the regularizing contribution $\left.\delta\left|\nabla_{x} \mathbf{u}\right|^{r-2} \nabla_{x} \mathbf{u}\right)$. Note that in particular the weak continuity of $\frac{1}{2}|\mathbf{u}|^{2}+e$ prescribed in (2.1) is a consequence of (1.37).

REMARK 5.1. In order to achieve the total energy balance (1.40) it is crucial to remove first the Galerkin approximation (otherwise the kinetic energy contribution is projected on the finite-dimensional space $\left.X_{N}\right)$. For this reason we need that, after taking the limit $N \rightarrow \infty$, we still have sufficient regularity to use $\mathbf{u}$ as a test function in the momentum equation in order to get a kinetic energy equality. This regularity is properly provided by the additional term $\delta\left|\nabla_{x} \mathbf{u}\right|^{r-2} \nabla_{x} \mathbf{u}$.

5.4. The limits $\varepsilon \rightarrow 0$ and $\delta \rightarrow 0$. After taking the limit $N \rightarrow \infty$, we still have to remove the regularizations coming from the parameters $\varepsilon$ (appearing in the coefficient $[\Gamma(\vartheta)]_{\varepsilon}$ in (4.4)), and $\delta$ (appearing in the mollification of the initial data, in the function $U_{\delta}$ in the approximation $\mathbb{H}_{\delta}$ of $\mathbb{H}$, and in the regularizing terms $[\mathbf{u}]_{\delta} \otimes \mathbf{u}$ and $\delta\left|\nabla_{x} \mathbf{u}\right|^{r-2} \nabla_{x} \mathbf{u}$ in the momentum equation).

Since the total energy balance (1.40) is already achieved and no quadratic terms are present in its right hand side, letting $\varepsilon \rightarrow 0$ and subsequently $\delta \rightarrow 0$ does not give rise to any additional difficulty. Actually, most of the argument can be carried out just by adapting the procedure, based on the uniform a priori bounds of Section 3, used before to let $m, N \rightarrow \infty$. The only point which requires some additional care 
is letting $\delta \rightarrow 0$ in the momentum equation. However, this procedure is absolutely analogous to the argument outlined in [8, Section 5.2], to which we refer the reader for details. We just note that we need here the restriction $r<10 / 3$ (cf. Section 4.1) on the exponent of the additional viscosity term (up to this point we only used that $r>3)$.

\section{REFERENCES}

[1] J.P. Aubin, Un théorème de compacité, (French) C. R. Acad. Sci. Paris, 256, 5042-5044, 1963.

[2] J.M. Ball and A. Majumdar, Nematic liquid crystals: From Maier-Saupe to a continuum theory, Mol. Cryst. Liq. Cryst., 525, 1-11, 2010.

[3] A.N. Beris and B.J. Edwards, Thermodynamics of Flowing Systems, Oxford University Press, Oxford, 1994.

[4] E. Bonetti, P. Colli, and M. Frémond, A phase field model with thermal memory governed by the entropy balance, Math. Models Meth. Appl. Sci., 13, 1565-1588, 2003.

[5] E. Bonetti and M. Frémond, A phase transition model with the entropy balance, Math. Meth. Appl. Sci., 26, 539-556, 2003.

[6] P.G. De Gennes and J. Prost, The Physics of Liquid Crystals, Oxford University Press, Oxford, 1974.

[7] C. Denniston, J.M. Tóth, and G. Yeomans, Lattice Boltzmann simulations of liquid crystal hydrodynamics, Phys. Rev. E, 63:056702, 2001.

[8] E. Feireisl, M. Frémond, E. Rocca, and G. Schimperna, A new approach to non-isothermal models for nematic liquid crystals, Arch. Rat. Mech. Anal., 205, 651-672, 2012.

[9] E. Feireisl and J. Málek, On the Navier-Stokes equations with temperature-dependent transport coefficients, Diff. Equ. Nonlin. Mech., Art. ID 90616, 14 (electronic), 2006.

[10] E. Feireisl and A. Novotný, Singular limits in thermodynamics of viscous fluids, Birkhauser, Basel, 2009.

[11] E. Feireisl, E. Rocca, and G. Schimperna, On a non-isothermal model for nematic liquid crystals, Nonlin., 24, 243-257, 2011.

[12] M. Frémond, Non-Smooth Thermomechanics, Springer-Verlag, Berlin, 2002.

[13] A. Friedman, Partial Differential Equations of Parabolic Type, Prentice-Hall, Englewood Cliffs, NJ, 1964.

[14] P. Krejčí, E. Rocca, and J. Sprekels, A nonlocal phase-field model with nonconstant specific heat, Interfaces Free Bound., 9, 285-306, 2007.

[15] N.V. Krylov, Boundedly nonhomogeneous elliptic and parabolic equations in a domain, (in Russian), Izv. Akad. Nauk SSSR, Ser. Mat., 47, 75-108, 1983.

[16] N.V. Krylov and M.V. Safonov, A property of the solutions of parabolic equations with measurable coefficients, (Russian) Izv. Akad. Nauk SSSR Ser. Mat., 44, 161-175, 1980.

[17] O.A. Ladyzhenskaya, V.A. Solonnikov, and N.N. Uralceva, Linear and Quasilinear Equations of Parabolic Type, AMS, Trans. Math. Monograph 23, Providence, 1968.

[18] J.L. Lions, Quelques méthodes de Résolution des Problèmes aux Limites Non Linéaires, Dunod, Gauthier-Villars, Paris, 1969.

[19] W. Maier and A. Saupe, A simple molecular statistical theory of the nematic crystalline-liquid phase, Z. Naturf., 14a, 882-889, 1959.

[20] N. Mottram and C. Newton, Introduction to Q-Tensor Theory, University of Strathclyde, Mathematics Research Report, 10, 2004.

[21] L. Nirenberg, On elliptic partial differential equations, Ann. Scuola Norm. Sup. Pisa (3), 13, 115-162, 1959.

[22] M. Paicu and A. Zarnescu, Global existence and regularity for the full coupled Navier-Stokes and Q-tensor system, SIAM J. Math. Anal., 43, 2009-2049, 2011.

[23] M. Paicu and A. Zarnescu, Energy dissipation and regularity for a coupled Navier-Stokes and Q-tensor system, Arch. Rat. Mech. Anal., 203, 45-67, 2012.

[24] R.T. Rockafellar, Convex Analysis, Princeton Mathematical Series, Princeton University Press, Princeton, N.J., 28, 1970.

[25] N. Schopohl and T.J. Sluckin, Defect core structure in nematic liquid crystals, Phys. Rev. Lett., $59,2582-2584,1987$. 